Standke, Jan

\title{
Gegenwartsliteraturforschung - Gegenwartsliteraturunterricht? Thomas Glavinics Romane in literaturwissenschaftlicher und deutschdidaktischer Perspektive
}

Standke, Jan [Hrsg.]: Die Romane Thomas Glavinics. Literaturwissenschaftliche und deutschdidaktische Perspektiven. Frankfurt, M. : Lang 2014, S. 19-51. - (Beiträge zur Literatur- und Mediendidaktik; 25)

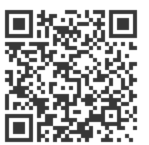

Quellenangabe/ Reference:

Standke, Jan: Gegenwartsliteraturforschung - Gegenwartsliteraturunterricht? Thomas Glavinics

Romane in literaturwissenschaftlicher und deutschdidaktischer Perspektive - In: Standke, Jan [Hrsg.]:

Die Romane Thomas Glavinics. Literaturwissenschaftliche und deutschdidaktische Perspektiven.

Frankfurt, M. : Lang 2014, S. 19-51 - URN: urn:nbn:de:0111-pedocs-128971 - DOI: 10.25656/01:12897

https://nbn-resolving.org/urn:nbn:de:0111-pedocs-128971

https://doi.org/10.25656/01:12897

in Kooperation mit / in cooperation with:

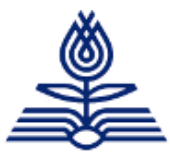

\section{PETER LANG}

INTERNATIONALER VERLAG DER WISSENSCHAFTEN

http://www.peterlang.com

\section{Nutzungsbedingungen}

Gewährt wird ein nicht exklusives, nicht übertragbares, persönliches und beschränktes Recht auf Nutzung dieses Dokuments. Dieses Dokument ist ausschließlich für den persönlichen, nicht-kommerziellen Gebrauch bestimmt. Die Nutzung stellt keine Übertragung des Eigentumsrechts an diesem Dokument dar und gilt vorbehaltlich der folgenden Einschränkungen: Auf sämtlichen Kopien dieses Dokuments müssen alle Urheberrechtshinweise und sonstigen Hinweise auf gesetzlichen Schutz beibehalten werden. Sie dürfen dieses Dokument nicht in irgendeiner Weise abändern, noch dürfen Sie dieses Dokument für öffentliche oder kommerzielle Zwecke vervielfältigen, öffentlich ausstellen, aufführen, vertreiben oder anderweitig nutzen.

Mit der Verwendung dieses Dokuments erkennen Sie die Nutzungsbedingungen an.

\section{Terms of use}

We grant a non-exclusive, non-transferable, individual and limited right to using this document.

This document is solely intended for your personal, non-commercial use. Use of this document does not include any transfer of property rights and it is conditional to the following limitations: All of the copies of this documents must retain all copyright information and other information regarding legal protection. You are not allowed to alter this document in any way, to copy it for public or commercial purposes, to exhibit the document in public, to perform, distribute or otherwise use the document in public.

By using this particular document, you accept the above-stated conditions of use.

\section{Kontakt / Contact:}

peDOCS

DIPF | Leibniz-Institut für Bildungsforschung und Bildungsinformation

Informationszentrum (IZ) Bildung

E-Mail: pedocs@dipf.de

Internet: www.pedocs.de

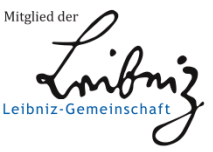


Jan Standke (Hrsg.)

Die Romane

Thomas Glavinics

Literaturwissenschaftliche und deutschdidaktische Perspektiven

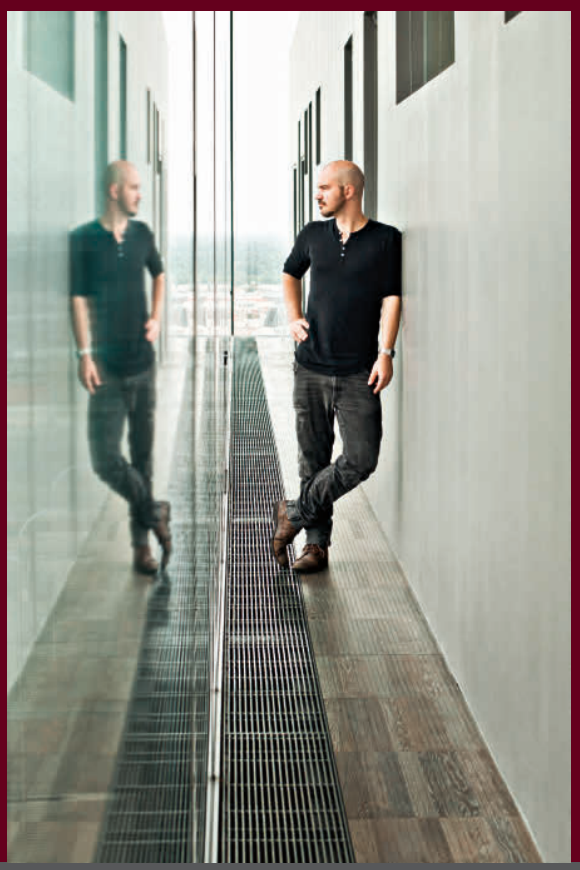




\section{Jan Standke (Hrsg.)}

\section{Die Romane Thomas Glavinics}

Seit Erscheinen der Erfolgsromane Der Kameramörder (2001) und Die Arbeit der Nacht (2006) gehört Thomas Glavinic zu den wichtigen Stimmen der deutschsprachigen Gegenwartsliteratur. In seinen Romanen erzählt er auf immer wieder neue Weise von Welten und Figuren, die dem Leser gleichermaßen vertraut und bedrohlich fremd erscheinen. Die Beiträge des Bandes erschließen die facettenreiche und vielfach ausgezeichnete Prosa des Autors aus zwei sich ergänzenden Perspektiven: Im ersten Teil des Bandes sind literaturwissenschaftliche Einzelanalysen der Romane sowie Studien zu den poetologischen Texten versammelt. Der zweite Teil bietet literatur- und mediendidaktische Beiträge, die sowohl in theoretischer wie auch konkret methodischer Absicht nach der Bedeutung von Glavinics Werk für den Deutschunterricht fragen. Ein ausführliches Interview mit Thomas Glavinic leitet den Band ein.

\section{Der Herausgeber}

Jan Standke ist Juniorprofessor für Fachdidaktik Deutsch an der Universität Magdeburg. 
Die Romane Thomas Glavinics 


\title{
BEITRÄGE ZUR LITERATUR- UND MEDIENDIDAKTIK
}

\author{
Herausgegeben von \\ Bodo Lecke und Christian Dawidowski
}

BAND 25 
Jan Standke (Hrsg.)

\section{Die Romane Thomas Glavinics}

Literaturwissenschaftliche und deutschdidaktische Perspektiven 


\title{
Bibliografische Information der Deutschen Nationalbibliothek
}

Die Deutsche Nationalbibliothek verzeichnet diese Publikation in der Deutschen Nationalbibliografie; detaillierte bibliografische Daten sind im Internet über http://dnb.d-nb.de abrufbar.

\author{
Umschlagabbildung: \\ Thomas Glavinic. \\ (C) Flora P. Photography, Graz. \\ www.florap.com
}

\author{
ISSN 1617-531X \\ ISBN 978-3-631-63899-6 (Print) \\ E-ISBN 978-3-653-04917-6 (E-Book) \\ DOI 10.3726/978-3-653-04917-6 \\ (C) Peter Lang GmbH \\ Internationaler Verlag der Wissenschaften \\ Frankfurt am Main 2014 \\ Alle Rechte vorbehalten. \\ Peter Lang Edition ist ein Imprint der Peter Lang GmbH. \\ Peter Lang - Frankfurt am Main · Bern · Bruxelles · \\ New York · Oxford $\cdot$ Warszawa $\cdot$ Wien
}

Das Werk einschließlich aller seiner Teile ist urheberrechtlich geschützt. Jede Verwertung außerhalb der engen Grenzen des

Urheberrechtsgesetzes ist ohne Zustimmung des Verlages

unzulässig und strafbar. Das gilt insbesondere für

Vervielfältigungen, Übersetzungen, Mikroverfilmungen und die Einspeicherung und Verarbeitung in elektronischen Systemen.

Diese Publikation wurde begutachtet.

www.peterlang.com 


\title{
Gegenwartsliteraturforschung - Gegenwartsliteraturunterricht? Thomas Glavinics Romane in literaturwissenschaftlicher und deutschdidaktischer Perspektive
}

\author{
Jan Standke
}

Für M. \& S., meine Liebsten

\section{Gegenwartsliteraturforschung?}

Die Frage, was unter deutschsprachiger, Gegenwartsliteratur' zu verstehen, wie mit ihr methodisch angemessen umzugehen - kurz: wie über sie ,zu sprechen“ sei, ist für die germanistische Literaturwissenschaft traditionellerweise problematisch. Mit verschiedenen - sich in der Fachgeschichte jedoch mitunter wiederholenden Argumenten - wurde die Beschäftigung mit der neuesten Literatur in der Vergangenheit deshalb nicht selten an den Rand des Faches delegiert oder ganz der Literaturkritik überantwortet. Seit einigen Jahren hat die Gegenwartsliteratur im Fachdiskurs jedoch wieder auf breiterer Basis an Attraktivität gewonnen. Dokumentiert wird das Interesse durch Forschungsnetzwerke, zahlreiche Konferenzen und Sammelbände. ${ }^{1}$ Nicht zuletzt spielt die Gegenwartsliteratur, wie die Lehrveranstaltungsverzeichnisse deutscher Universitäten zeigen, auch im germanistischen Studium und damit in Teilen der Deutschlehrerbildung wieder vermehrt eine Rolle.

Überblickt man vor diesem Hintergrund die germanistischen Debatten zur Bedeutung und Funktion der Gegenwartsliteratur in den letzten Jahrzehnten, so zeigt sich - wie einleitend bereits angemerkt -, dass dieses Engagement der Germanistik keineswegs konstant war. Der aktuellen Phase literaturwissenschaftlicher Aufmerksamkeit gingen Perioden voraus, die von einem konjunkturell deutlich schwankenden Interesse an der jeweils neuesten Literatur geprägt waren. ${ }^{2}$ Den Rahmenbedingungen dieser wechselhaften und bislang noch weithin ungeschriebenen Geschichte der literaturwissenschaftlichen Auseinandersetzung mit dem Begriff der, literarischen Gegenwart' und den ihm jeweils zugeordneten Autoren und Texten widmet sich mittlerweile eine von der modernen Wissenschaftsforschung inspirierte Fachgeschichte der Literaturwissenschaft.

1 Vgl. z.B. Bierwirth / Johannsen / Zeman (Hrsg.) 2012; Brodowsky / Klupp (Hrsg.) 2010; Eichhorn (Hrsg.) 2014; Horstkotte / Herrmann 2013.

2 Vgl. zur Fachgeschichte der germanistischen Literaturwissenschaft unter besonderer Berücksichtigung des Umgangs mit Gegenwartsliteratur Eke 2012; Braun 2010. 
Gefragt wird dabei nach dem „literaturwissenschaftlichen doing things with text“, das durch „Wertungs- und Zuschreibungsprozesse [bestimmt ist], die durch Wiederholung und Routine“ ein implizites Wissen herausbilden und verfestigen, das in bestimmte Teilzeiträumen der germanistischen Fachgeschichte wiederum die Praktiken im literaturwissenschaftlichen Feld beeinflusst. Neben der Etablierung dieser wissenschaftssoziologischen Perspektive wurde im Rahmen der jüngeren Diskussion noch auf einen weiteren blinden Fleck des literaturwissenschaftlichen Interesses am literarischen Feld der Gegenwart hingewiesen:

Wenig ausgeprägt ist bislang außerdem die Bereitschaft der Wissenschaftler, den Praktiken und Techniken anderer Akteure, die am ,Gegenwartsliteratur-Machen' beteiligt sind - namentlich jener des Literaturbetriebs -, entsprechende Aufmerksamkeit zu schenken. Insbesondere vor dem Hintergrund des massiven Wandels im literarischen Feld der letzten Jahrzehnte - man denke etwa an aktuelle Konzentrationsprozesse im Buchhandel und Verlagswesen, an die gewachsene Relevanz von Literaturvermittlungsformaten wie Lesungen und Festivals oder an die Etablierung der Creative-Writing-Schulen - scheint es aber geboten, Texte verstärkt in ihren Kontexten zu lesen, sprich: die Literatur im Zusammenhang ihrer sozialen, kulturpolitischen und ökonomischen Entstehungs-, Distributions- und Rezeptionsbedingungen wahrzunehmen. Aufgrund der ökonomischen, medialen und institutionellen Neuerungen im literarischen Feld müsste Literatur auch als kulturelle Praxis und soziales Handeln in institutionalisierten Rollen verstanden werden. Demgemäß gilt es, das gesamte literarische Feld, vor allem auch den Literaturbetrieb, in den Blick zu nehmen. ${ }^{3}$

Momentan zeichnen sich, so könnte man die hier entworfene sozialgeschichtlich informierte Programmatik zusammenfassen, mehr und mehr die Konturen einer ,Gegenwartsliteraturforschung' $\mathrm{ab}$, die durch die begriffliche und methodische Reflexion sowie die (Rück-)Gewinnung kontextualisierender Sichtweisen vermutlich langfristig - ,historisch begründeten Berührungsängste bezüglich des Forschungsgegenstandes ,Gegenwartsliteratur“" ${ }^{4}$ überwunden $\mathrm{zu}$ haben scheint.

Dass ein solcher, auf die literarische Gegenwart gerichteter Diskurs seine ,Vorzeigeautoren' kürt, an deren Schaffen sich die neuen Lesarten in exemplarischer Weise besonders eindringlich entfalten und erproben lassen, ist nicht verwunderlich. Unter diesen Autoren, denen sich die neue ,Gegenwartsliteraturforschung' bevorzugt widmet, nimmt der österreichische Autor Thomas Glavinic einen prominenten Platz ein. Kaum ein aktueller literaturwissenschaftlicher Sammelband zur Gegenwartsliteratur, der das Euvre und die mediale Selbstin-

3 Bierwirth / Johannsen / Zeman 2012, S. 12.

4 Ebd., S. 9. 
szenierung des Autors nicht berücksichtigt. ${ }^{5}$ Indem Glavinics Texte - vom literarischen Debüt Carl Haffners Liebe zum Unentschieden (1998) bis zum monumentalen und die Reihe der Jonas-Romane vorläufig abschließenden Roman Das größere Wunder (2013) - auf so unvergleichliche und immer wieder neue Weise „leichtfüßig von der Schwere“ erzählen, ${ }^{6}$ eröffnen sie der literaturwissenschaftlichen Forschung einen analytisch schwerlich auszuschöpfenden literarischen Kosmos. In diesem ausufernden inhaltlichen und formalen Reichtum erkennt Andrea Bartl jedoch auch einen roten Faden, der sich durch das Werk des Österreichers zieht: „Glavinics Texte erarbeiten in ihren Figurendarstellungen die anthropologische Diagnose, der Mensch sei beständig von Angst, Einsamkeit und dem Tod bedroht, was in ihm aber, neben Verzweiflungs- oder ApathieSchüben, ,trotzdem' eine paradoxe Suche nach dem Glück und einer utopischen Vorstellung von Liebe auslöst." " Glavinics Romane sezieren somit sprachgewaltig die Existenzbedingungen der Gegenwartsgesellschaft und offenbaren dabei treffsicher sowohl die Abgründe des (post-)postmodernen Subjekts als auch dessen Techniken der ,Selbstsorge ${ }^{6}$

Aber nicht nur Glavinics Romane sind für die Gegenwartsliteraturforschung von Interesse. Auch die vielfältigen Inszenierungspraktiken der „Subjektform Autor" - gemeint sind ,jene paratextuellen und habituellen Techniken und Aktivitäten von Schriftstellern, mit denen sie öffentlichkeitsbezogen für ihre eigene Person, für ihre Tätigkeit und / oder für ihre Produkte Aufmerksamkeit erzeugen (wollen) ${ }^{\text {‘9 }}$ - sind Thema zahlreicher jüngerer Untersuchungen. $\mathrm{Zu}$ solchen „Techniken und Aktivitäten“ gehört beispielsweise das Format der Poetikvorlesung. ${ }^{10}$ Glavinic liefert der Gegenwartsliteraturforschung auch hier reiches Material, an dem sich der neue analytische Blickwinkel erproben lässt. Im Sommer 2012 nahm er die renommierte Poetikprofessur der Universität Bamberg wahr. In öffentlichen Vorträgen und Seminaren gab er Einblicke in seine Poetologie

5 Vgl. Braun 2010, S. 15f.; Johannsen 2011; Assmann 2011.

6 Bartl 2014, S. 14.

7 Ebd.

8 Auf Glavinics Romane soll im Rahmen dieser einführenden Überlegungen nicht näher eingegangen werden. Die ausführliche inhaltliche Auseinandersetzung bleibt den Beiträgen des vorliegenden Bandes vorbehalten. Da die meisten der hier veröffentlichten Beiträge bei Erscheinen des letzten Romans, Das größere Wunder (2013), bereits in der Endphase bzw. abgeschlossen waren, wird der umfangreichste Text Glavinics nur am Rande behandelt.

9 Jürgensen / Kaiser 2014, S. 220. Vgl. hierzu auch dies. 2011 und Grimm / Schäfer 2007. Vgl. hierzu auch die Beiträge von Walter-Jochum, Hillebrandt, Dörfelt-Mathey, Pottbeckers und Hofer im vorliegenden Band.

10 Vgl. hierzu Hachmann 2014. 
und berichtete über sein Verhältnis zu professionellen Lesern. ${ }^{11}$ Gegenüber der literaturkritischen Wertung und literaturwissenschaftlichen Deutung seiner Texte ,inszeniert ${ }^{\star}$ - um die Terminologie der Gegenwartsliteraturforschung beizubehalten - sich Glavinic als aufgeschlossener Autor:

Ich mag Menschen, die ihr Leben der Literatur widmen und sich mit Respekt und Beharrlichkeit in Romane hineinarbeiten. Es gibt Kritiker, die ich nicht mag, weil es ihnen nicht um die Literatur geht, sondern nur um sich selbst, um ihre Karriere oder ihr Ego. Ich mag Kritiker, die sachlich und zugleich leidenschaftlich für die Literatur arbeiten. Ich mag Literaturwissenschaftler. Ich mag sie besonders, wenn sie Literatur wirklich lieben. Was ich sehr mag, sind kluge Menschen, speziell solche, die über meine Bücher schreiben. In den Zeitungen liest man tiefergehende Betrachtungen zu einem Roman ja selten, weil da zum einen zu wenig Platz ist für eine substanziellere Auseinandersetzung mit einem Buch und weil Rezensenten zum anderen offenbar unter großem zeitlichen und ökonomischen Druck stehen, weswegen sie Bücher zunehmend querzulesen scheinen. So etwas mag ich nicht. Ich mag es, wenn Menschen über meine Romane nachdenken. ${ }^{12}$

Als Glavinic im Rahmen eines Kolloquiums im Anschluss an die Poetikprofessur mit wissenschaftlichen Vorträgen über sein Werk konfrontiert wurde, ging es dem Autor dann aber trotz aller Offenheit doch ein wenig zu weit: „Die Vorträge über meine Literatur [...] habe ich mir vor Ort nicht angehört. Eine Weile versuchte ich es, doch es machte mich ziemlich nervös. ${ }^{\text {“13 }}$ Der Dialog zwischen Autor und Gegenwartsliteraturforschung brach wegen Glavinics Flucht in den sonnigen Garten der Bamberger Villa Concordia jedoch nicht ab, sondern setze sich in vielen Einzelgesprächen produktiv fort. Interessanterweise trifft auf Glavinics Verhältnis zur Literaturwissenschaft in diesem Zusammenhang zu, was in ganz ähnlicher Weise über eine vergleichbare Veranstaltung mit seinem Landsmann Thomas Bernhard 35 Jahre zuvor resümierend vermerkt wurde:

Und Bernhard selbst? Er war immerhin gekommen, hatte vorgelesen und einen Abend lang mitangehört, wie man seine Geistesarbeit klinisch zerlegte [...]. Danach blieb er den Ausführungen und Diskussionen fern. Aber er zeigte sich, so wortkarg er auch in der Öffentlichkeit auftrat, dennoch Gesprächen im kleinen Kreis und in informeller Umgebung keineswegs abgeneigt, ja, schien erfreut, [...] Leser und Freunde zu finden. Der Einfall [...], einen Autor in engen Kontakt mit seinen Interpreten und beide zugleich vor ein Publikum zu bringen, hatte sich gerade im Fall Thomas Bernhards - fast möchte man sagen, überraschend - bewährt. ${ }^{14}$

11 Bartl / Glasenapp / Hermann 2014.

12 Glavinic 2014a, S. 27.

13 Glavinic 2014b, S. 12.

14 Spiel 1977. 
Der vorliegende Band möchte den Dialog zwischen Literaturwissenschaft bzw. Literatur- und Mediendidaktik, dem Autor Thomas Glavinic und seinem Werk weiterführen, ausweiten und intensivieren, aber auch - nicht zuletzt in methodischer Hinsicht - auf die Probe stellen. Die Ausgangsbedingungen für dieses Vorhaben sind günstig. Die Pfade zum Werk des Österreichers sind geebnet, aber noch längst nicht ausgetreten: Der Tagungsband zur Bamberger Poetikprofessur liefert zahlreiche Impulse, die sich für eine vertiefte Beschäftigung mit Glavinics Literatur dankbar aufgreifen lassen. Mit der Veröffentlichung der Poetikvorlesungen unter dem Titel Meine Schreibmaschine und ich (2014) liegen nunmehr auch die poetologischen Reflexionen des Autors in verdichteter Form vor, die eine weiterführende Auseinandersetzung mit dem publizierten Werk stimulieren können. Weiterhin wurden in den zurückliegenden Monaten - wie weiter oben bereits angeführt - zahlreiche Beiträge veröffentlicht, die das ,Autorensubjekt ${ }^{*}$ Glavinic im Praxiszusammenhang des literarischen Betriebs der Gegenwart untersuchen, womit den eingangs offengelegten Desiderate der literaturwissenschaftlichen Analyse des literarischen Feldes bereits in konkreten Studien begegnet wird.

Neben der Fortführung der in ersten Ansätzen bereits etablierten literaturwissenschaftlichen Forschung zum Werk Thomas Glavinics möchten die Beiträge der zweiten Sektion des vorliegenden Bandes das Perspektivenspektrum im Umgang mit der Gegenwartsliteratur noch erweitern. Denn eine Durchsicht der rezenten Forschung lässt einen weiteren ,blinden Fleck ${ }^{`}$ zutage treten. Auch wenn in der Gegenwartsliteraturforschung verstärkt nach den Bedingungen und Verlaufsformen der Produktion, Distribution und Rezeption neuester Literatur gefragt wird, bleibt doch ein wichtiger Vermittlungskontext der Gegenwartsliteratur weithin unberücksichtigt: der schulische Deutschunterricht. Für diesen Problemzusammenhang - Gegenwartsliteratur und Deutschunterricht - ist traditionellerweise die Literaturdidaktik zuständig. In den zurückliegenden Jahren hat die Literaturdidaktik ihr Gesicht verändert. Die ,empirische Wende' der Disziplin führte dazu, dass die theoretische Modellierung und praktische Überprüfung von Kompetenzen sprachlich-literarischen Verstehens und Lernens unter immer neuen gesellschaftlich evozierten Vorzeichen - aktuell etwa der Inklusion - in den Vordergrund rückten. Dass die Fachdidaktik auf diese Weise den tatsächlichen Prozessen des sprachlich-literarischen Lernens unter den Bedingungen sich verändernder Voraussetzungen in Schule und Gesellschaft näher kommt, ist ohne Zweifel ein Gewinn. Es zeigt sich aber auch, dass die Gegenstände des literarischen Lernens - die literarischen Texte und andere ästhetische Medien - zuweilen zugunsten des empirischen Diskurses aus dem Blick geraten. Auf die Frage „Wo bleibt die Literatur?“ lässt sich aus literaturdidaktischer Sicht - sieht man von der weithin florierenden Forschung zur Kinder- und Ju- 
gendliteratur einmal ab - zurzeit keine vollkommen befriedigende Antwort geben. Die Beiträge des Bandes wollen am Beispiel der Romane Thomas Glavinics theoretische wie auch konkret methodische Perspektiven eines ,Gegenwartsliteraturunterrichts' skizzieren, der vertieftes literarisches Lernen im Umgang mit anspruchsvollen Texten anbahnt und im übergreifenden Medienkontext entfaltet. ${ }^{15}$ Durch das in diesem Band realisierte Nebeneinander literaturwissenschaftlicher und literatur- und mediendidaktischer Beiträge, hierbei setzt sich das Konzept von anderen Publikationen zur deutschsprachigen Gegenwartsliteratur ab, soll zudem deutlich werden, dass zwischen Literaturwissenschaft und didaktik gerade im Sinne einer Gegenwartsliteraturforschung zahlreiche Schnittpunkte existieren, die mit Blick auf eine gelingende, fachwissenschaftliche und fachdidaktische Inhalte gleichermaßen zur Geltung bringende germanistische Lehrerbildung fruchtbare Kooperationen ermöglichen können. Um den weiteren Kontext der Diskussion um Gegenwartsliteratur im Deutschunterricht noch etwas weiter zu erhellen, sollen die Eckpunkte der literaturdidaktischen Debatten im Folgenden knapp rekonstruiert und mit Blick auf den Aspekt der ,Identitätsorientierung' des Literaturunterrichts enggeführt werden. Am konkreten Beispiel der Romane Thomas Glavinics werden abschließend einige Möglichkeiten der Behandlung im Unterricht angedeutet, die von den literatur- und mediendidaktischen Beiträgen des Bandes dann weiterentwickelt und konkretisiert werden.

\section{Gegenwartsliteraturunterricht? ${ }^{16}$}

In der Literaturdidaktik besteht grundsätzlich ein allgemeiner Konsens über die Bedeutung der Gegenwartsliteratur für das literarische Lernen im Deutschunterricht. In den bundesweit geltenden Bildungsstandards für das Fach Deutsch wie auch in den jeweiligen Lehrplänen der einzelnen Bundesländer ist Gegenwartsliteratur ebenfalls kein Fremdwort mehr. Und dennoch: Umfassende und systematische Überlegungen zu einer Didaktik der Gegenwartsliteratur stellen nach wie

15 Vgl. hierzu auch Standke 2014c.

16 Die folgenden Überlegungen basieren auf einem Vortrag, der im Rahmen des wissenschaftlichen Kolloquiums zur Poetikprofessur Thomas Glavinics gehalten und in überarbeiteter Form auch im zugehörigen Tagungsband veröffentlicht wurde (Standke 2014). Ich danke den Organisatoren und Teilnehmern des Kolloquiums für das Interesse an den literaturdidaktischen Überlegungen, die mich darin bestärkt haben, den Dialog zwischen Literaturwissenschaft und Deutschdidaktik auch im Zusammenhang des vorliegenden Bandes weiterzuführen. 
vor die Ausnahme dar. ${ }^{17}$ Eine ähnliche Zurückhaltung lässt sich auch mit Blick auf die Unterrichtspraxis konstatieren. Tatsächlich wird im Unterricht nur wenig neueste Literatur behandelt. Die Literaturdidaktik hat für diese Abstinenz verschiedene Erklärungen angeboten. So ist die mangelnde Experimentierfreudigkeit der Lehrer ${ }^{18}$ bei der Textauswahl ein häufig formulierter Vorwurf. ${ }^{19}$ Elisabeth Paefgen beurteilt den Stellenwert der Gegenwartsliteratur im Deutschunterricht deshalb skeptisch: „Groß dürfte die ,Gefahr der Aktualität“ in der Praxis des Literaturunterrichts wahrscheinlich nicht sein; es gibt unter den Deutschlehrern eine Tendenz zum alten und neuen Kanonischen." ${ }^{20}$ Dass die hier benannte ,Tendenz', im Unterricht auf Bewährtes zurückzugreifen, keinesfalls als grundsätzliche Verweigerung des Deutschunterricht der Gegenwartsliteratur gegenüber zu begreifen ist, zeigen die jüngeren Unterrichtsmodelle und -vorschläge in den deutschdidaktischen Zeitschriften - vor allem im Bereich der Kinder- und Jugendliteratur, ${ }^{21}$ das Interesse der Lehrer im Rahmen von Fortbildungsveranstaltung und natürlich nicht zuletzt die Beiträge des vorliegenden Bandes. Dennoch ist Paefgens Diagnose freilich nicht verfehlt. Die Gegenwartsliteratur stellt einen „Problemfall literarischer Sozialisation“ dar. ${ }^{22}$ Es ist deshalb dringend erforderlich, den literaturdidaktischen Diskurs über die Bedeutung neuester Literatur im Unterricht fortzuführen und auszudehnen.

Für das ,Experiment' Gegenwartsliteratur im Deutschunterricht soll im Weiteren am Beispiel der Romane Thomas Glavinics plädiert werden. Hierfür werden zunächst knapp die wesentlichen Linien der literaturdidaktischen Diskussion um die Chancen und Risiken neuester Literatur im Unterricht nachgezeichnet. Es wird dann zu diskutieren sein, welche Bedeutung den Romanen Thomas Glavinics mit Blick auf ein zentrales Ziel des Deutschunterrichts - der „Förderung der Identitätsentwicklung “23 ${ }^{62}$ der Schüler - zukommen kann. Die problematischen Charaktere, von denen die Romane bevölkert sind, müssen eine massive Hürde für einen Unterricht darstellen, der die Förderung der Identitätsentwicklung der Schüler vorrangig an die lesende Begegnung mit positiven Helden bindet. Demgegenüber soll herausgestellt werden, dass sich die Chancen eines ,identitätsorientierten` Umgangs mit Gegenwartsliteratur gerade aus den Mög-

17 Vgl. Dawidowski 2012.

18 Vgl. Kammler/Surmann 2000.

19 Mit der männlichen Form sind im gesamten Beitrag wie auch bei ,Schüler' selbstverständlich stets beide Geschlechter gemeint.

20 Paefgen 2006, S. 94.

21 Vgl. z.B. Wrobel 2013, Dawidowski 2012, Standke 2013, Standke 2014c.

22 Kammler 2001.

23 Vgl. Pfäfflin 2010, S. 44f. 
lichkeiten zur Konfrontation der jugendlichen Leser mit fremden und irritierenden Welten und Figuren ergeben.

\section{Wer ist ,ich‘?}

Den Ich-Erzähler in Thomas Glavinics Roman Das bin doch ich (2007), einen gleichnamigen jungen österreichischen Schriftsteller, umtreiben Sorgen, die den realen Autoren der deutschsprachigen Gegenwartsliteratur und nicht zuletzt dem realen Schriftsteller Glavinic keineswegs fremd sein dürften. Der Weg zu literarischem Erfolg und finanzieller Unabhängigkeit ist steinig. Mit ironischer Überzeichnung erzählt der Roman in zuweilen skurril-grotesken Episoden von den Erfahrungen eines jungen Autors mit den Instanzen und stereotyp konturierten Figuren des zeitgenössischen Literaturbetriebs. Die narrative Grundkonstruktion des Textes und das konsequent realistische Erzählen legen eine Lesart nahe, die ganz gezielt das Verhältnis von Textwelt und Wirklichkeit, das im Roman codierte „Weltwissen“ ${ }^{24}$ fokussiert. Von Anfang an ist die Wirkung von Das bin doch ich durch die schon im Titel angelegte autofiktionale Spannung zwischen dem realen Autor Thomas Glavinic und seinem Ich-Erzähler bestimmt. Der Text zwingt seine Leser damit regelrecht zu einer ,doppelten' Lektüre, die einerseits der fiktionalen Handlung des Romans zu folgen hat, andererseits beständig der vorgeblich autobiographischen Selbstreferenzialität des Erzählens auf den Leim zu gehen droht. ${ }^{25}$

24 Vgl. Abraham / Launer (Hrsg.) 2002.

25 In Interviews weist Glavinic auf die Fiktionalität der Romanhandlung hin: „Ich weiß nicht, wie oft ich noch betonen muss, dass Das bin doch ich ein Roman ist! Und keine Autobiografie!“ (Meine Dämonen spuken zu Hause. Interview mit Thomas Glavinic, 05.09.2009, in: http:/www.diepresse.com/home/kultur/literatur/506409/Thomas-Glavinic_Meine-Daemonenspuken-zu-Hause). Anders verhält es sich hingegen mit Unterwegs im Namen des Herren. Der literarischen Reportage (2011) bescheinigt der Autor ausdrücklich einen autobiographischen Kern: „Unterwegs im Namen des Herrn ist kein Roman, der Ich-Erzähler mit mir identisch. [...] Wichtig war mir daran die Position des Erzählers, und die ist nicht neu. Auch Journalisten sind Menschen. Der eine sieht dies, der andere jenes. Jeder Text ist gefärbt von den persönlichen Erfahrungen seines Verfassers, von seinem Charakter, seinen Interessen, ja seiner augenblicklichen Befindlichkeit. Ich stehe auf dem Standpunkt, dass ich Informationen darüber, über den Berichterstatter, im Text mitliefern muss, damit der Leser besser einzuschätzen vermag, was er vom Gelesenen halten darf. Ich schreibe anders über einen konservativen Wallfahrtsort als, sagen wir, der von mir sehr geschätzte Martin Mosebach. Also hat der Leser ein Anrecht auf Information über mich, es muss Schlaglichter auf meine Persönlichkeit geben, damit der 
Nachdem im letzten Drittel des Romans die Veröffentlichung des neuesten Werks, Die Arbeit der Nacht, endlich gesichert ist, verfällt der Ich-Erzähler in einen Zustand bedrückender „Leere“ - sowohl ökonomischer als auch kreativproduktiver Art. Die verbleibende Zeit bis zur Veröffentlichung des Romans gerät für den fiktiven Glavinic daher zur Keimzelle des Zweifels:

Ich sehe überall schlechte Vorzeichen, oder anders: Ich bin zweckpessimistisch und fühle mich schon durch Kleinigkeiten in meiner Sorge bestätigt. Gestern kam die Verlagsschau. Sieht gut aus, aber ich fand natürlich sofort Grund zur Beunruhigung. Bei einigen Autoren wird gleichzeitig das Hörbuch ausgeliefert, bei mir nicht. [...] Das gefällt mir nicht. Aber egal, selbst wenn mich Michael Krüger morgen anruft und sagt, bis auf einen Kerl in Greifswald lieben alle Kritiker mein Buch, werde ich verzweifelt sein, denn dieser Jemand in Greifswald ist das Böse unter der Sonne. (2007: 178)

Die zunächst noch aufgeschobene Eingliederung des Romans in das lukrative Medienverbundsystem und die literaturkritische Würdigung seiner Texte stellen für Glavinic wichtige Indikatoren des literarischen Erfolgs dar. Hinzu treten im Verlauf der Romanhandlung allerlei weitere normative Instanzen, deren Urteil sich der Ich-Erzähler zumeist unfreiwillig ausliefert. An ihnen muss sich nicht nur das neue Werk, sondern auch Glavinic als Schriftstellerpersönlichkeit und Mensch messen lassen: Da ist zum Beispiel der geschätzte Autorenkollege Daniel Kehlmann. In unzähligen Anrufen und SMS lässt er Glavinic an der Erfolgsgeschichte seines jüngst veröffentlichten Buchs Die Vermessung der Welt teilhaben. Des Weiteren erleben wir Glavinic in Gesprächen mit seiner Agentin, anderen Schriftstellern und Kulturschaffenden, aber auch der eigenen Familie. Nicht zuletzt die Figur der Mutter des fiktiven Autors lenkt den Blick mit unsensibler Direktheit immer wieder auf jene Gratifikationen, die den Autoren der Gegenwartsliteratur Autorität und wirtschaftliche Sicherheit verschaffen, z.B. die Nominierung für die Shortlist des Deutschen Buchpreises. Das System des Literaturbetriebs samt seiner Erfolgserwartungen durchzieht die erzählte Welt bis in den letzten Winkel. Selbst die mütterliche Anerkennung ist vom ,Dichterruhm' des Sohnes keineswegs unabhängig.

In den zahlreichen Bezügen zum aktuellen Literaturbetrieb, die in Das bin doch ich in ironischer Weise inszeniert werden, erkennen Literaturkritik und die in ihrer Programmatik weiter oben charakterisierte Gegenwartsliteraturforschung den bedeutsamen Beitrag des Romans zur deutschsprachigen Gegenwartsliteratur. Als so genannter neuerer „Literaturbetriebsromans ${ }^{\text {‘26 }}$ ist der Text

Leser entscheiden kann, inwieweit er mir vertraut. Aus meiner Sicht ist dies der wichtigste handwerkliche Aspekt dieses Buches." Glavinic 2014b, S. 88.

26 Vgl. zur Klassifizierung von Das bin doch ich als Literaturbetriebsroman Braun 2010, S. 15f.; Johannsen 2011; Assmann 2011. 
deshalb bereits in einen Kanonisierungsprozess involviert, der auch Autoren wie Martin Walser, Klaus Modick, John von Düffel oder Wolfgang Hegewald einschließt. ${ }^{27}$ Die Selbstbespiegelung des Literaturbetriebs gehört zum festen Themenrepertoire der Gegenwartsliteratur. Interessant zu beobachten ist dabei, mit welchen Mitteln die Autoren Distanz schaffen zwischen ihren Figuren und der eigenen Stellung im literarischen Feld. Doch bei aller Affinität zur ironischen Überzeichnung, die für dieses Genre typisch ist, eröffnen solche Texte, wie bereits angedeutet, auch den Durchgriff auf außertextuelle Wirklichkeit. Die inszenierten Unwägbarkeiten des Wegs zu literarischer Bedeutsamkeit und Nachruhm verweisen auf die nüchternen Entsprechungen in der Welt des zeitgenössischen Literaturbetriebs. Mit Blick auf Skandale wie beispielsweise den um Martin Walsers Roman Tod eines Kritikers (2002) wird deutlich, wie sich gerade im Literaturbetriebsroman die permanent unzuverlässige Verschiebung der Grenze zwischen Fiktionalität und Wirklichkeit als konstitutives Wirkungsprinzip manifestiert. $^{28}$

Deshalb kann es durchaus sinnvoll sein, ganz im Sinne des erweiterten Erkenntnisinteresses der Gegenwartsliteraturforschung, diese Romane auf ihr spezifisches ,Betriebswissen“ hin zu befragen: Welche Bedingungen müssen erfüllt sein, damit sich ein Text in der unüberschaubaren Vielfalt der literarischen Gegenwart zumindest kurzfristig durchzusetzen vermag - oder gar im besten Fall: einen Platz im kulturellen Gedächtnis der Gesellschaft besetzen kann? Glavinic und die oben angeführten Autoren geben auf diese Frage recht unterschiedliche Antworten. ${ }^{29}$ Statt die verschiedenen Sichtweisen auf mögliche Strategien und Wege zum schriftstellerischen Ruhm hier im Einzelnen zu entfalten und auf ihren Wirklichkeitsgehalt hin zu befragen, soll der Blick stattdessen nochmals auf einen weiteren blinden Fleck des erzählten Literaturbetriebs gelenkt werden. Es ist nämlich interessant zu beobachten, was diese Texte im Nachdenken über die Bedingungen von Wirkung und Bestand der Literatur im kulturellen Bewusstsein weithin ausblenden oder doch nur ganz am Rande erwähnen: den Deutschunterricht.

Die Abwesenheit des Deutschunterrichts bzw. des Bildungsraums Schule in den neueren Romanen über den Literaturbetrieb - hier decken sich die blinden Flecken in Literatur und Literaturwissenschaft gewissermaßen - bedeutet nun aber keineswegs, dass literarische Bildung und Sozialisation in diesen Texten

27 Vgl. Walser 2002; Modick 2006; Hegewald 2009; von Düffel 2011. Hierzu auch Braun 2012, S. 9ff.

28 Vgl. hierzu Borchmeyer / Kiesel (Hrsg); Braun 2012, S. 96-109; Hinkerohe 2006; Hofer 2007; Neuhaus 2004.

29 Vgl. hierzu bes. die aufschlussreichen Bemerkungen Glavnics im Rahmen der Bamberger Poetikvorlesung, Glavnic 2014b . 
keine Rolle spielen. Ganz im Gegenteil: In Das bin doch ich beispielsweise stößt der Leser wiederholt auf Spuren literarischer Bildungsprozesse. Sie finden sich dort, wo von prägenden Leseerfahrungen und literarischen Präferenzen erzählt wird: Exemplarisch hierfür sind die Affinität des fiktiven Glavinic zu Karl May, seine Begeisterung aus Anlass der Lektüre von Denis Johnsons Traindreams oder die Erinnerung an beglückende Kindheitserlebnisse mit einem Klassiker der Kinder- und Jugendliteratur. In Das bin doch ich kommt es z.B. während einer Zugfahrt nach Graz zu einer Situation, die ein Schlaglicht auf die Bedeutung literarischer Bildung wirft:

Vielleicht liegt es am Alkohol, aber das ist unwichtig, jedenfalls bin ich glücklich dieses Buch gekauft zu haben und es jetzt lesen zu können. Dieses Gefühl ist etwas Konstantes und Kostbares in meinem Leben, ich kenne es, seit ich sieben Jahre alt war und unter dem Weihnachtsbaum mit Huckleberry Finn anfing. Ich sitze im Zug, lese Traindreams und fühle mich geborgen, ich habe mehr als ein Buch, mehr als einen Gegenstand gekauft, ich habe mir Gedanken gekauft, die Chance, mehr zu werden. (2007: 159)

Sich emphatisch ganz in die Lektüre von Literatur versenken zu können, aus ihr Vergnügen, Entlastung und Wohlbefinden, aber auch Weltwissen, Ideen und Impulse für die Formung der eigenen Identität zu beziehen, dies alles sind Ziele einer literarischen Bildung, wie sie in unterschiedlichen gesellschaftlichen Kontexten, vorzugsweise aber im Deutschunterricht erworben werden kann. ${ }^{30}$ In Das bin doch ich ereignet sich ein weiterer Schlüsselmoment literarischer Sozialisation im familiären Umfeld - unter dem Weihnachtsbaum. Den ,Leser' Thomas Glavinic prägt diese Erfahrung bis ins Erwachsenenalter. Darüber, wie sich das Interesse und die Begeisterung für Literatur im Bildungsraum Schule weiterentwickelt haben, wird hingegen nichts erzählt. Das mag zunächst durchaus verwundern. Gehört es doch sonst zum Inventar schriftstellerischer Selbstreflexion, Leseerfahrungen im Bildungsraum Schule - ganz gleich, ob sie als hemmend oder förderlich erfahren worden sind - in der autobiographischen Selbstbesichtigung aufzugreifen und für die eigene literarische Entwicklung zu bewerten.

Aber auch unabhängig vom Blick auf die Bedingungen der Förderung des schriftstellerischen Vermögens spielen die Institutionen der Bildung in Das bin doch ich keine hervorgehobene Rolle. Ob eines seiner Werke einmal Gegenstand des schulischen Literaturunterrichts sein wird oder sein sollte, scheint dem Glavinic des Romans jedenfalls nicht sonderlich bedenkenswert zu sein. Warum eigentlich nicht? Der Deutschunterricht stellt heutzutage schließlich oftmals den Raum bereit, in dem Kinder und Jugendliche vergleichsweise intensive und leider nicht selten die einzigen Erfahrungen mit anspruchsvollen literarischen Tex- 
ten machen. Die Präferenzen zukünftiger Leser oder auch Nichtleser von Gegenwartsliteratur werden im Deutschunterricht mitgeprägt. Allein schon deshalb wäre es für erfolgsorientierte Autoren wie den fiktiven Glavinic des Romans nicht verfehlt, die Schule als wichtige Instanz der Literaturvermittlung und kanonisierung stärker in Betracht zu ziehen. Daniel Kehlmann - hier ließe sich die amüsante Konkurrenzgeschichte der Romanhandlung fortspinnen - hat bereits Einzug in die schulischen Lehrpläne gehalten. Dass Die Vermessung der Welt ihre Stellung im Deutschunterricht auch langfristig behaupten wird, ist unter anderem daran zu erkennen, dass alle größeren Schulbuchverlage mittlerweile zahlreiche Erläuterungen und Unterrichtshandreichungen auf den Markt geworfen haben. ${ }^{31}$ Für die Romane Glavinics stand eine solche breit angelegte didaktische Aufbereitung bislang noch aus. Die fachdidaktischen Beiträge im vorliegenden Band schicken sich an, dieses Desiderat anzugehen. Das dürfte zumindest dem Glavinic des Romans nicht egal sein.

Das Genre des neueren Literaturbetriebsromans hat einen festen Platz im Textrepertoire der deutschsprachigen Gegenwartsliteratur. Wie sich bislang zeigte, kommt zumindest dieses Sujet ohne den Deutschunterricht ganz gut aus. Klammert man das Feld der Kinder- und Jugendliteratur aus, ${ }^{32}$ so lässt sich diese Einschätzung sicherlich auch für den Gesamtbereich der Gegenwartsliteratur verallgemeinern. ${ }^{33}$ Wie verhält es sich nun umgekehrt? Kommt man auch in der Schule ohne den Literaturbetriebsroman oder - ebenfalls allgemeiner gefragt ohne die Gegenwartsliteratur aus? Eine Antwort auf diese Frage muss - wie eingangs angedeutet - ambivalent ausfallen. Von der Relevanz aktueller Texte für das literarische Lernen im Deutschunterricht ist man zwar überzeugt. Literaturdidaktiker wie Clemens Kammler bemühen sich seit Jahren um die stärke Berücksichtigung der literarischen Gegenwart im Deutschunterricht in ihrer ganzen Breite. ${ }^{34}$ Tatsächlich werden aber nach wie vor nur wenige und zumeist dieselben Texte behandelt. Der Trend, die Textauswahl am ,Bewährten` zu orientieren, gilt für die Literatur anderer Zeiträume natürlich in gleicher Weise. ${ }^{35}$

31 Vgl. z.B. Nadolny 2012; Hellberg 2012.

32 Dass das Thema Schule und Unterricht in der Kinder- und Jugendliteratur eine wichtige Rolle spielt, sollte angesichts der Adressierung und Intention der entsprechenden Texte kaum überraschen, vgl. Zabka 2008.

33 Ausnahmen bestätigen freilich auch hier die Regel. Vgl. etwa Schalansky 2011; Orths 2003.

34 Vgl. Kammler 1995.

35 Vgl. Paefgen 2006, S. 94; Kammler 1995, S. 129. 


\section{Deutschunterricht und die (Dauer-)Baustelle Gegenwartsliteratur}

Die Randständigkeit der Gegenwartsliteratur im Deutschunterricht hat ihre Gründe. Trotz der vergleichsweise intensiven literaturdidaktischen Auseinandersetzung mit neueren Texten und Aktualisierungstendenzen in Lehrwerken, ${ }^{36}$ haben der Deutschunterricht und die Literaturdidaktik auf die Herausforderung der Gegenwartsliteratur insgesamt bislang noch keine überzeugenden Antworten gefunden. Ohne die Diskussion um die Rolle der Gegenwartsliteratur im Deutschunterricht hier im Einzelnen und im Detail nachzeichnen zu können, lässt sich doch in groben Zügen das Folgende festhalten: Man tut sich in Universität und Schule schwer mit der neuesten deutschsprachigen Literatur. ${ }^{37}$ Diese Diagnose selbst ist wiederum keineswegs neu. ${ }^{38}$ Vielmehr begleitet sie die Entwicklung der Literaturwissenschaft und Literaturdidaktik seit über 80 Jahren. ${ }^{39}$ Ablesbar sind diese Schwierigkeiten schon an den Titeln einschlägiger Publikationen. Da ist die Rede von der Baustelle Gegenwartsliteratur, ${ }^{40}$ von Maulhelden und Königskindern ${ }^{41}$ oder von der Folgenlosigkeit neuester Texte im Literaturunterricht. ${ }^{42}$

Vor dem Hintergrund bildungspolitischer Reformen eröffneten sich in den 1980er und 1990er Jahren Spielräume in den schulischen Lehrplänen, die hauptsächlich in der Sekundarstufe II einen Einbezug neuester, vor allem erzählender Literatur ermöglichten. Demgegenüber nahm die Aufmerksamkeit für die Schreibweisen der Gegenwart ${ }^{43}$ in den folgenden Jahren wieder merklich ab. Mitte der 1990er Jahre sprach man im literaturdidaktischen Kontext bereits vom Experiment Gegenwartsliteratur, ${ }^{44}$ wenig später von Gegenwartslücken. ${ }^{45}$ Die mit der Semantik des Experiments implizierte Unvorhersehbarkeit des Verlaufs und der Ergebnisse des literarischen Lernens mit und an Gegenwartstexten, bringt die damaligen Vorbehalte vieler Lehrkräfte sicherlich angemessen auf den Punkt. Daran hat sich bislang wenig geändert. Für den neuerlichen Geltungsverlust lassen sich verschiedene Ursachen ausmachen. Die wichtigsten seien hier nur stichwortartig genannt: ${ }^{46}$

36 Vgl. z.B. Dreier 2005; Pfäfflin 2010.

37 Vgl. aber auch Huber / Meier (Hrsg.) 2010.

38 Vgl. Kämper-van den Boorgaart 2004.

39 Vgl. bereits Schönbrunn 1929. Siehe hierzu Frank 1977, S. 729-748.

40 Erb (Hrsg.) 1998.

41 Köhler (Hrsg.) 1998.

42 Fingerhut 1988.

43 Schumacher 2011.

44 Vgl. Kammler 1995; Kammler/ Surmann 2001; Kammler 2002.

45 Kammler 1998.

46 Vgl. zum Folgenden Dawidowski 2012. 
Entscheidend für die stärkere Berücksichtigung der Gegenwartsliteratur in den 1980er und 1990er Jahren waren die Diskussionen um Postmoderne und Poststrukturalismus. Neben den damaligen Bemühungen, aus den philosophischen Debatten methodische Konsequenzen für den literaturwissenschaftlichen Umgang mit Texten des Kanons zu ziehen, ${ }^{47}$ rückten auch die Literatur und Kunst der Gegenwart mit ihren neuen Verfahren des Erzählens und Darstellens als Ausdrucksmedien einer neuen Sichtweise auf die Wirklichkeit in den Blick. Seit sich die entsprechenden Debatten in der Bezugsdisziplin Literaturwissenschaft merklich abgekühlt bzw. auf andere Problemstellungen verlagert haben, ist auch das Interesse an der Literatur der Gegenwart wieder ein Stück weit geschwunden. Für Verunsicherungen und Zurückhaltung vieler Deutschlehrer gegenüber der neuesten deutschsprachigen Literatur dürften auch die heftigen Auseinandersetzungen über Anspruch und Qualität der Gegenwartsliteratur gesorgt haben, die sich Autoren, Literaturkritiker, Verleger und Lektoren in den 1990er Jahren lieferten. ${ }^{48}$ Wenn es in der Literaturkritik hauptsächlich scharfe Verrisse literarischer Neuerscheinungen hagelte, ist es nicht verwunderlich, wenn Lehrer angesichts der ohnehin knapp bemessenen Lesezeit lieber auf Bewährtes zurückgriffen. Von nicht unerheblicher Bedeutung für die Einstellung vieler Lehrer dürfte außerdem die Erfahrung des damaligen Germanistikstudiums gewesen sein, in dem weithin die Ausrichtung der Lehre am historischliterarischen Kanon dominierte.

Mehr als 10 Jahr später sind die großen Debatten über die Gegenwartsliteratur verklungen. Stattdessen erlebt der ,Literaturskandal' ein Revival als Ereignis literarischer Kommunikation. ${ }^{49}$ Einfacher geworden ist die Orientierung im literarischen Feld aber für Lehrer auch im Jahr 2014 nicht. Im Gegenteil. Angesichts der großen Menge an literarischen Neuerscheinungen stehen Deutschlehrer der Forderung, die Clemens Kammler 1995 formuliert hat, zumeist ratlos gegenüber: „Wir Deutschlehrer und Deutschlehrerinnen müssen uns der Unübersichtlichkeit des Literaturmarktes stellen, ständig nach für die Schule Brauchbarem Ausschau halten, ohne gleich den Kanon im Hinterkopf zu haben. Dabei gibt es keine Garantie dafür, daß die Texte, auf die wir dabei stoßen, in zehn Jahren für die Schule noch interessant sind. “50 Hier ist die Literaturdidaktik gefragt, den Markt kritisch zu sondieren und Auswahlprozesse für den Unterricht gezielt zu begleiten und zu unterstützen. Sieht man vom Bereich der Kinder- und Jugendliteratur ab, finden sich seitens der Literaturdidaktik bezüglich

47 Vgl. z.B. Bossinade 2000. Für den literaturdidaktischen Bereich vgl. z.B. Bark / Förster (Hrsg.) 2000; Kammler 2000.

48 Vgl. hierzu die Beiträge in Köhler / Moritz 1998.

49 Vgl. Friedrich (Hrsg.) 2009; Neuhaus / Holzner (Hrsg.) 2007.

50 Kammler 1995, S. 129. 
literarischer Neuerscheinungen jedoch vermehrt skeptische Urteile. Es mangelt an Texten, die inhaltliche Attraktion, konzeptionelle Komplexität und schülergerechte Lesbarkeit miteinander verbinden. ${ }^{51}$ Die Literatur, die in den letzten Jahren wegen ihres provozierenden Gehalts oder ihrer Konventionen überschreitenden, innovativen Form Aufmerksamkeit erregt hat, eignet sich in der Regel nur sehr bedingt für den Einsatz im Deutschunterricht. ${ }^{52}$ Vor diesem Hintergrund ist es nicht verwunderlich, dass sich die literarische Gegenwart des Deutschunterrichts auf bewährte und unterrichtsmethodisch ausführlich erschlossene Texte wie Patrick Süßkinds Das Parfum, ${ }^{53}$ Bernhard Schlinks Der Vorleser oder Robert Schneiders Schlafes Bruder verengt. Nicht zuletzt mag der Geltungsverlust der lange Zeit als Chiffre der literarischen Gegenwart geltenden neueren Popliteratur einschließlich ihrer medialen Präsenz dazu beigetragen haben, dass neueste Texte wieder an die Peripherie des deutschdidaktischen Diskurses gerückt sind. Wenn in der Lektüreliste für das niedersächsische Zentralabitur 2013/14 Christian Krachts Faserland (1995) und Florian Illies Generation Golf (2000) unter dem Schlagwort „Neue und neueste Tendenzen der Erzählliteratur“ erscheinen, so handelt es sich insgesamt besehen zweifellos um einen Vorstoß des Deutschunterrichts in die literarische Gegenwart. Strenggenommen bilden die hier gewählten Texte die Lebenswelt der Schüler jedoch schon nicht mehr ab und sind der ,Gegenwart' deshalb bereits entrückt. ${ }^{54}$

Bei den bislang angeführten Aspekten handelt es sich in der Hauptsache um Spezifika des literarischen Feldes. Möchte man das Verhältnis von Gegenwartsliteratur und Deutschunterricht bzw. Literaturdidaktik angemessen beurteilen, darf man freilich nicht außer Acht lassen, dass der für die jüngsten Entwicklungen entscheidende Einschnitt auch ein Ergebnis bildungspolitischer Entscheidungen ist. Die Zentralisierung und Kompetenzorientierung des Deutschunterrichts hat sich nicht nur auf die Bedeutung der Gegenwartsliteratur im Deutschunterricht im Besonderen, sondern auch auf den Status literarischer Bildung im Allgemeinen ausgewirkt. ${ }^{55}$ In der Folge von PISA und anderen Vergleichsstudien, die sich mit den grundlegenden sprachlichen Fähigkeiten von Schülerinnen und Schülern beschäftigen, haben sich die Prioritäten im schulischen Bildungskonzept verschoben. An die Stelle traditioneller Bildungsinhalte und Lernpro-

51 Bogdal 2004.

52 Vgl. Dawidowski 2012, S. 3.

53 Vgl. zur kanonischen Stellung des Romans im Deutschunterricht kritisch Bogdal 1993.

54 Vgl. zur Periodisierungsproblematik der Gegenwartsliteratur Braun 2012, S. 9-37; Bluhm 2007, S. 267.

55 Vgl. hierzu aktuell Wintersteiner 2011; siehe auch Abraham / Bremerich-Vos / Frederking / Wieler (Hrsg.) 2003; Kammler (Hrsg.) 2006; Rösch (Hrsg.) 2008; Rösch (Hrsg.) 2010 . 
zesse - so kann man es bei den Kritikern der Kompetenzorientierung nachlesen - sei eine ministeriell verordnete und ökonomisch durchdrungene Verwertungslogik getreten, die den Deutschunterricht auf das Training von konkret messbaren sprachlichen und rezeptionsbezogenen Fähigkeiten beschränkt. ${ }^{56}$ Diese Entwicklungen muss man ernst nehmen. Der Blick auf die Wirklichkeit des Studiums und des Literaturunterrichts sollte jedoch nicht durch überspitzte Oppositionen verstellt werden. In der konkreten Praxis der Lehrerbildung an den Hochschulen und Universitäten gilt die Neuorientierung des Deutschunterrichts in ihrer verabsolutierten Variante vielerorts als problematisch. Und auch in den Schulen sollte man zwischen dem Unterrichtsalltag und den auf spezifische Kompetenzen abzielenden Leistungsmessungen unterscheiden. Dennoch zeichnet sich aber die Tendenz ab, dass die zentralen Vorgaben und Anforderungen für einen kompetenzorientierten Deutschunterricht zu einer inhaltlichen Verknappung führen. Das könnte dem Literaturunterricht, der die nachhaltige Förderung von Lesekompetenz und -motivation erst durch vielfältige und lebensweltnahe Lektüreangebote sicherstellen kann, langfristig gefährlich werden. Dass Deutschlehrer angesichts dieser Entwicklungen eher auf das Bewährte zurückgreifen als sich dem Experiment zu öffnen, lässt sich daher durchaus nachvollziehen.

Vor diesem Hintergrund über die Perspektiven literarischer Bildung im Umgang mit Gegenwartsliteratur nun ausgerechnet am Beispiel der Romane Thomas Glavinics nachzudenken, könnte - wie eingangs bereits angedeutet - riskant sein. Und dies nicht nur, weil die Romane mit ihrer anspruchsvollen poetologischen Konzeption und ihrem Umfang die Möglichkeiten der Schüler und des Literaturunterrichts auf die Probe stellen. Auch wenn man die Identitätsentwürfe betrachtet, die in Glavinics Texten vermittelt werden, könnte sich hinsichtlich der Ziele des Deutschunterrichts Widerspruch regen. Lässt sich die Literatur der Gegenwart mit dem Anspruch des Deutschunterrichts, Schüler in der individuellen Identitätsarbeit zu fördern, in Einklang bringen? Dieser Aspekt scheint in besonderer Weise begründungsbedürftig zu sein. Schließlich erscheinen Identitäten in der Gegenwartsliteratur oftmals nicht mehr als homogene und in sich geschlossene Persönlichkeitsentwürfe, die den Rezipienten ohne Weiteres einen

56 Die Debatte um die Kompetenzorientierung des Deutschunterrichts soll an dieser Stelle nicht im Einzelnen nachgezeichnet werden. Vgl. hierzu die in Fußnote 55 angegebene Literatur. Zugespitzt hat sich die Kontroverse um den Stellenwert der literarischen Bildung zuletzt im Jahr 2011 in einer Debatte in der Zeitschrift Didaktik Deutsch. Mehrere Literaturdidaktiker reagierten auf Werner Wintersteiners Rede „Alte Meister - Über die Paradoxien literarischer Bildung“ (S. 5-22). Die einschlägigen Positionen und Argumente der Diskussion lassen sich hier im Zusammenhang nachvollziehen. Vgl. dazu auch Standke 2013. 
identifikatorischen Zugang ermöglichen. ${ }^{57}$ Selbst wenn die radikalen Tendenzen der narrativen Identitätsdissoziation, wie sie für die postmoderne Literatur typisch waren, ${ }^{58}$ im Erzählen der Gegenwart teilweise zurückgenommen werden, inszenieren neueste Texte keinesfalls die Rückkehr des homogenen Subjekts. ${ }^{59}$ Wird die Identitätsproblematik in Das bin doch ich letztlich vor allem als literaturtheoretische Frage nach dem Zusammenspiel von Autor und Ich-Erzähler verhandelt, präsentieren andere Romane Glavinics ungleich schwierigere Identitätsentwürfe. Der sich mehr und mehr selbst verlierende Jonas in Die Arbeit der Nacht ${ }^{60}$ Das Leben der Wünsche ${ }^{61}$ und schließlich Das größere Wunder, der zunächst so vertraut-unscheinbare, dann psychopatisch-kriminelle Lebenspartner in Der Kameramörder ${ }^{62}$ der durch abweichendes Verhalten auffallende Karl Kolostrum in Wie man leben soll, ${ }^{63}$ der bis zur Selbstvernichtung introvertierte Carl Haffner in Carl Haffners Liebe zum Unentschieden ${ }^{64}$ und schließlich der vom Verfolgungswahn gepeinigte und im Drogen- und Alkoholrausch delierende Protagonist in Lisa ${ }^{65}$ Es gelingt diesen Figuren kaum, die prekären Situationen, denen sie sich ausgesetzt sehen bzw. in die sie sich selbst manövrieren, zu bewältigen. Das Scheitern ist in Glavinics Euvre stets wahrscheinlicher als der Gewinn, die Chance, dem Schicksal eine positive Wendung zu verleihen, zumeist gering. Die Gefahr für die Figuren, die Stabilität der eigenen Identität zu verlieren, ist groß, dieser Gefahr zu unterliegen, der Normalfall. Glavinics Romane im Deutschunterricht - also eher ein Beitrag zur ,Identitätsgefährdung‘?

\section{Identitätsorientierung und literarische Bildung}

Die kritische Nachfrage erscheint zunächst berechtigt. Eine der zentralen Aufgaben des Deutschunterrichts besteht schließlich darin, Schüler durch den Umgang mit Sprache und Literatur in der Entwicklung und Ausprägung ihrer Persönlichkeit unterstützend zu begleiten. So wird etwa in den Bildungsstandards des Fachs Deutsch für den mittleren Schulabschluss der analytische wie auch handelnd-produktive Umgang mit Sprache, literarischen Texten und Medien im

57 Vgl. zum Begriff der Identität und den Praktiken der Identiätsarbeit in der Spät- bzw. Postmoderne Reckwitz 2006; Keupp u.a. 2008.

58 Vgl. Zima 2001.

59 Vgl. Neuhaus 2009.

60 Glavinic 2006.

61 Glavinic 2009.

62 Glavinic 2001.

63 Glavinic 2004.

64 Glavinic 1998.

65 Glavinic 2011. 
Wesentlichen dem Ziel einer positiven Entwicklung der Identität der Schüler untergeordnet:

Die Heranwachsenden lernen, auf der Grundlage der mit den fachlichen Inhalten und Methoden vermittelten Werte- und Normvorstellungen gesellschaftlichen Anforderungen zu begegnen, Lebenssituationen sprachlich zu bewältigen, sich mitzuteilen - zu argumentieren, Gefühle und Vorstellungen sprachlich zu fassen -, Kritikfähigkeit zu entwickeln, Leseerfahrungen zu nutzen und in kritischer Distanz zwischen Lebenswirklichkeit und den in Literatur und Medien dargestellten virtuellen Welten zu unterscheiden. Dies dient wesentlich der Persönlichkeitsentwicklung, das heißt der Stärkung von Selbstbewusstsein, Sozialkompetenz und Teamfähigkeit. ${ }^{66}$

Überlegungen, wie die Auseinandersetzung mit literarischen Texten im Deutschunterricht vor dem Hintergrund eines modernen, sozialphilosophisch und anthropologisch begründeten Identitätsbegriffs förderlich auf die Entwicklung der Schülerpersönlichkeit einwirken kann, finden in der Literaturdidaktik verstärkt seit den 1970er Jahren statt. Hervorzuheben ist in diesem Zusammenhang Jürgen Krefts grundlegende und vielbeachtete Studie Grundprobleme der Literaturdidaktik, in der er sich ausführlich mit der Bedeutung des literarischen Lernens für die Identitätsentwicklung als Selbst- und Weltverhältnis auseinandergesetzt hat. ${ }^{67}$ An seinem 4-Phasen-Modell der Literaturaneignung ${ }^{68}$ lässt sich die Zentralstellung des schülerbezogenen Identitätsaspekts im Akt des Textverstehens deutlich ablesen. Auch Henriette Herwig argumentiert bezüglich des Aspekts des Fremdverstehens für eine gezielte Entwicklung identitätsfördernder Kompetenzen im Rahmen der Auseinandersetzung mit Literatur im Unterricht. ${ }^{69}$ Sie weist dabei ausdrücklich darauf hin, dass die Arbeit an der eigenen Identität nicht ausschließlich durch das Erfahren des Bekannten und Vertrauten zu begleiten sei. Stattdessen bezieht sie die Konfrontation mit dem Fremden und Unbekannten in produktiver Absicht ausdrücklich in ihre Konzeption ein. Neben der

66 Sekretariat der Ständigen Konferenz der Kultusminister der Länder in der Bundesrepublik Deutschland 2003, S. 6.

67 Kreft 1977.

68 Kreft entwickelt in seinen Grundproblemen der Literaturdidaktik ein Phasenmodell, das für die literaturdidaktische Theoriebildung wie auch für die Unterrichtspraxis bis in die Gegenwart von Bedeutung ist. Er unterscheidet mehrere Phasen voneinander, die das Verhältnis des Schülers zum Text und zu sich selbst verschiedentlich organisieren: die Phase der bornierten Subjektivität, die Phase der Objektivierung, die Phase der Aneignung und reflektierten Subjektivität sowie die Phase der Applikation. Im Sinne einer Synthese von Schüler- und Sachorientierung weist er darauf hin, dass ,,[d]as adäquate Verstehen poetischer Texte [...] in vier Hauptphasen [verläuft]. In allen Phasen sind alle Momente enthalten: Interpretation und Applikation, Subjektivität und Kreativität usw., aber unterschiedlich akzentuiert". (S. 379f.)

69 Vgl. Herwig 1980. 
Entwicklung einer auf das literarästhetische Lesen wie auch auf die pragmatische Kommunikation in der Lebenswelt gerichteten Fähigkeit zur Empathie, spielen somit auch die Kompetenz, Fremdes auszuhalten und zu eingespielten Sichtweisen auf Distanz zu gehen eine wesentliche Rolle. Volker Frederking beschreibt die Zielstellung der mehr oder weniger gelenkten Konfrontation der Schüler mit fremden oder befremdlichen Inhalten und Perspektiven literarischer Texte folgendermaßen:

Ambiguitätstoleranz bzw. Rollendistanz sind die identitätsfördernden Lernpotentiale einer solchen Rezeptionserfahrung. Einerseits nämlich lernt das lesende Subjekt Widersprüchliches, Widerständiges bzw. Andersartiges zu ertragen, d.h. Ambiguität zu tolerieren. Andererseits ermöglicht das Einlassen auf textlich in Erscheinung tretendes Fremdes die Erfahrung der Rollendistanz bzw. die Vorbereitung auf diese. Denn wenn ich mich für etwas im lesenden Verstehen öffne, das meinem eigenen gewohnten Handlungs- und Denkrahmen zuwiderläuft, distanziere ich mich probehandelnd. $^{70}$

Kaspar H. Spinner hat den Fokus der Identitätsorientierung vom Prozess des Textverstehens dann auch auf den Akt des literarischen Schreibens ausgeweitet. ${ }^{71}$ Der Ausdruck in der Schriftsprache erfüllt im Rahmen der Identitätsentwicklung eine wichtige Funktion, indem im Zusammenspiel von personalem Schreib- und sich anschließendem Leseprozess ,Selbstobjektivation“ ermöglicht wird. Identitätsorientierter Literaturunterricht im Zusammenhang von Textverstehen, literarischem Schreiben und ästhetischem Erleben geht somit über die genaue Analyse eines Textes hinaus. Vielmehr soll es darum gehen, einen Raum zu eröffnen, in dem die Schüler spezifische ästhetische Erfahrungen machen können - „die genießende Reflexion einer fremden Gedankenwelt und die Möglichkeit eines durch Reflexion und Kommunikation veränderten Bezugs zu sich und zu anderen". 72

Es ist nicht verwunderlich, dass die Identitätsorientierung des Deutschunterrichts im Zeichen einer verstärkten Pluralisierung, aber auch Individualisierung und Medialisierung von Gesellschaft und Lebenswelt zunehmend an Bedeutung gewinnt. ${ }^{73}$ Die Sozialisation von Kindern und Jugendlichen ist mehr und mehr durch heterogene Einflussfaktoren bedingt, wobei sich nationalkulturelle Räume zunehmend entgrenzen und durch virtuelle Erlebniswelten ergänzt werden. Literatur gilt in diesem Zusammenhang (noch) als bevorzugtes Medium der Identitätsentwicklung im Deutschunterricht. In Literatur sind Entwürfe von Identität sprachlich gestaltet. Sie bieten Schülern einen Raum für symbolisches Probe-

70 Frederking 2010, S. 423.

71 Vgl. Spinner 1980. Siehe auch ders. 2001.

72 Frederking 2010, S. 428.

73 Vgl. hierzu ausführlich ebd., S. 429ff. 
handeln in vertrauten Mustern, aber auch für Erfahrungen des historisch ${ }^{74}$ und kulturell Fremden. ${ }^{75}$ Figuren können in bedeutsamen Situationen der Identitätsfindung beobachtet und deren Entscheidungen auf eigene Bedürfnisse, Erfahrungen und Probleme bezogen werden.

Die Versuchung, literarische Texte danach auszuwählen, ob sie die Identitätsbildung von Schülern durch nachahmenswerte Figuren in positiver Weise unterstützen, ist nicht gering. Der identitätsorientierte Literaturunterricht hat deshalb bereits mehr als einmal unter „Therapieverdacht“ gestanden. ${ }^{76}$ Die Überzeugung, dass die Persönlichkeitsentwicklung durch positive Identitätsentwürfe besonders effektiv zu unterstützen sei, ist nach wie vor weit verbreitet. Wie oben gezeigt werden konnte, ist die Annahme in dieser Absolutheit aber nicht hinreichend begründet. Nicht zuletzt jedoch aufgrund der Vorbehalte gegen irritierende Identitätsentwürfe dominieren vor allem in der Sekundarstufe I Texte aus dem Bereich der Kinder- und Jugendliteratur, ${ }^{77}$ die bekanntermaßen eine große Menge positiver Helden bereithalten. In der Sekundarstufe II erfolgt hingegen weitgehend der Rückzug auf die literarischen Klassiker.

Indem gegen die verbreitete Präferenz für positive Helden der oben erläuterte Aspekt des notwendigen Zusammenspiels von Eigen- und Fremderfahrungen für die Entwicklung einer stabilen Identität nun wieder aufgriffen wird, kann demonstriert werden, dass sich Glavinics Romane mit Gewinn zum Gegenstand literarischer Bildungsprozesse im Deutschunterricht machen lassen. ${ }^{78}$ Gerade

74 Vgl. Brüggemann 2008.

75 Vgl. Dawidowski / Wrobel (Hrsg.) 2006.

76 Vgl. Frederking 2010, S. 424ff.

77 Vgl. Lange (Hrsg.) 2012; Gansel 2010.

78 Literarische Bildung und literarisches Lernen beziehe ich hier auf einen Vorschlag Kaspar Spinners. Mit Blick auf die Praxis des Literaturunterrichts stellt er die bislang überzeugendste Vermittlung von Kompetenzorientierung und literarischen Bildungszielen dar. In einem vielbeachteten Beitrag in der Zeitschrift Praxis Deutsch formulierte Spinner 2006 elf „Aspekte literarischen Lernens“. Seither wurden diese Teilfähigkeiten vielfach diskutiert, kritisiert und ergänzt, vgl. Vgl. Kammler 2006; Saupe 2007. Zu den Teilfähigkeiten literarischer Bildung gehören nach Spinner: „1. Beim Lesen und Hören Vorstellungen entwickeln, 2. Subjektive Involviertheit und genaue Wahrnehmung ins Spiel bringen, 3. Sprachliche Gestaltung aufmerksam wahrnehmen, 4. Perspektiven literarischer Figuren nachvollziehen, 5. Narrative und dramaturgische Handlungslogik verstehen, 6. Mit Fiktionalität bewusst umgehen, 7. Metaphorische und symbolische Ausdrucksweise verstehen, 8. Sich auf die Unabschließbarkeit des Sinnbildungsprozesses einlassen, 9. Mit dem literarischen Gespräch vertraut werden, 10. Prototypische Vorstellungen von Gattungen/Genres gewinnen, 11. Literaturhistorisches Bewusstsein entwickeln. “ Ergänzt wurde dieser Fähigkeitenkatalog u.a. von Abraham / Kepser 2009, S. 98f. Sie fordern, dass Schüler durch die Lektüre literarischer Texte auch Kritikfähigkeit sowie 
durch ihre irritierenden Figuren und erzählten Welten ermöglichen sie wichtige Perspektivwechsel, die für die kulturelle und soziale Teilhabe der Schüler an einer zunehmend pluralisierten und oftmals widersprüchlichen Lebenswelt unerlässlich sind. ${ }^{79}$

\section{Literatur- und mediendidaktische Perspektiven}

Thomas Glavinics Romane - dessen muss man sich freilich bewusst sein - sind keine leichte Kost. Schon gar nicht für Schüler, denen die literarische Lektüre durch die starken Konkurrenzangebote anderer Medien zunehmend verdächtig wird. Literatur bereitet Schwierigkeiten, von denen andere Medien entlastet sind. Welche Chancen kann da ein nahezu vierhundertseitiger Roman wie Die Arbeit der Nacht für einen Deutschunterricht bieten, der im Sinne der Entwicklung einer nachhaltigen Lesekultur die Förderung basaler Lesefähigkeiten ebenso ernst nimmt wie die Ziele literarischer Bildung. ${ }^{80}$ In der Sek. II, vorher würde man die Schüler mit einer solchen Textauswahl wohl überfordern, sieht sich der Deutschunterricht mit besonderen Schwierigkeiten konfrontiert. Nach wie vor dominieren hier die ,Klassiker' der Höhenkammliteratur. Die Distanz zu den Interessen und lebensweltlichen Problemen der Schüler lässt sich gerade unter der Zielstellung der Identitätsorientierung vielfach nur schwer überbrücken. Nun muss der Einsatz von Gegenwartsliteratur aber keineswegs zur Abkopplung von der literarischen Tradition führen, ganz im Gegenteil. Literaturhistorisches Bewusstsein zu fördern sollte heutzutage eben nicht ausschließlich bedeuten, nur

die Kompetenz, Literatur auf das in ihr aufgehobene Weltwissen hin zu befragen, erwerben sollten. Erst auf dieser Grundlage könne es gelingen, den Schülern die souveräne rezeptive wie auch produktive Teilhabe im Handlungsfeld Literatur zu ermöglichen. Als Kompetenzformulierungen sind diese Aspekte literarischen Lernens umstritten, da sie sich in vielen Fällen der empirischen Messbarkeit entziehen. Wie man die emphatische Versenkung eines Schülers in die Perspektive einer literarischen Figur genau bestimmen und zur Grundlage einer Kompetenzniveaubeurteilung machen kann, ist nicht geklärt. Dass dieser Aspekt für eine gewinnbringende Auseinandersetzung mit Literatur dennoch von grundlegender Bedeutung ist und dass man Aussagen darüber treffen kann, ob dies einem Schüler im Literaturunterricht zunehmend besser gelingt oder nicht, sollte hingegen klar sein. Vgl. auch die Erweiterungen und Präzisierungen des Modells literarischen Lernens, auf die Spinner kürzlich hingewiesen hat, Spinner 2013.

79 Freilich handelt es sich bei der Identitätsproblematik nicht um den einzigen Begründungszusammenhang, aus dem sich eine didaktische Auswahlentscheidung ableiten lässt. Wie im Weiteren zu zeigen ist, bieten Glavinics Texte ein weitaus größeres didaktisches Potential. Mir scheint aber die Debatte um die Identitätsorientierung des Deutschunterrichts zurzeit eine der maßgeblichen Hürden für Texte der Gegenwartsliteratur zu sein. 
von der geschichtlichen Dimension her zu denken. Es geht vielmehr darum, den Schülern zu verdeutlichen, dass auch ihre Welt der digitalen Gleichzeitigkeit das Ergebnis historischer Prozesse ist.

Konfrontiert man die Schüler mit der Grundsituation der Figur des Jonas aus Die Arbeit der Nacht, die sich schon aus der Lektüre der ersten Kapitel erschließt und daher einen vergleichsweise geringen Leseaufwand erfordert, so lässt sich leicht ein Netzwerk transmedialer Verweisungen erarbeiten: Ein in der modernen Welt einsames und isoliertes Individuum, das die Ursachen des Weltverlustes ergründen und seiner misslichen Lage entfliehen will - hierbei handelt es sich um ein ebenso klassisches wie aktuell vielfach inszeniertes Motiv. Harro Müller-Michaels spricht auch von einem anthropologisch aufgeladenen Denkbild, in dem sich menschliche Elementarerfahrungen in verdichteter Weise gestalten. ${ }^{81}$ Texte allein nach dem Kriterium ihrer ,Denkbildhaftigkeit‘ auszuwählen, ist sicherlich problematisch und wird zu Recht kritisiert. ${ }^{82}$ Man darf jedoch nicht unterschätzen, wie effektiv die Orientierung an solchen konzentrierten menschlichen Elementarerfahrungen die literarische Lektüre im Unterricht mit dem außerschulischen Rezeptionsverhalten und den Prozessen der Identitätsbildung der Schüler zu verbinden vermag. Die Vorliebe für fantastische Stoffe und Genres stellt eine wichtige Konstante in der außerschulischen Medienrezeption dar. Darauf wird seitens der Literaturdidaktik und Leseforschung hingewiesen. ${ }^{83}$ Ulf Abraham hat kürzlich die Eignung fantastischer Stoffe und Figuren für den Literaturunterricht erneut hervorgehoben und literaturdidaktisch ausführlich begründet. ${ }^{84}$ In den konkreten Auswahlentscheidungen für den Deutschunterricht wird dieser Aspekt aber noch zu wenig berücksichtig. Möchte man motivierend an die Interessen der Schüler anschließen, kann deshalb zuerst an erfolgreiche Filme gedacht werden, die sich der Gestaltung solcher anthropologischen Denkbilder in exemplarischer Weise widmen: Francis Lawrences' I am Legend (2007), Duncan Jones' Moon (2009) oder Robert Zemeckis Cast Away, um nur einige wenige Beispiele zu nennen. Fündig werden kann man ebenfalls im Bereich der Fernsehserien. Dehnt man das Motiv der Einsamkeit und Isolation auf Kollektive aus, kommen auch populäre Serien wie beispielsweise Star Gate Universe (2009-2011) oder Lost (2004-2010) als Vergleichsmedien in Frage. Die Behandlung von Die Arbeit der Nacht kann somit von den Vorerfahrungen der Schüler profitieren, an ihre Leseinteressen anknüpfen und den Einstieg in die vertiefte Textarbeit erleichtern.

81 Vgl. Müller-Michaels 1996.

82 Vgl. zu Begründungen der Auswahlkriterien von Gegenwartsliteratur Burtscher 2003; Pfäfflin 2010.

83 Vgl. z.B. Graf 2004.

84 Abraham 2012. Zum fantastischen Film vgl. auch Rauch 2011. 
Zunächst stellt die Konzeption des Romans die Schüler aber sicherlich vor einige Probleme. Nachdem die Grundsituation, in der sich Jonas befindet, umrissen ist, wendet sich das Geschehen ins Reflexive. Zahlreiche Erinnerungspassagen, in denen Jonas seiner schwindenden Selbstgewissheit neue Stabilität zu verleihen versucht, strukturieren die Handlung. Die hierdurch erzeugte Komplexität des Textes bei gleichzeitiger Entschleunigung der Handlung ist für Schüler nicht leicht zu bewältigen. Auch die sprunghaften Übergänge zwischen den einzelnen Handlungsteilen stellen an die Rezeptionsleistung der Schüler hohe Anforderungen. Der immer spontaner sich vollziehende Wechsel von Schlaf- und Wachzustand des Protagonisten erfordert zusätzliche Anstrengung im Prozess der Kohärenzbildung.

Eine didaktische Fokussierung auf das Moment der Identitätsspaltung kann die Bereitschaft zum genauen Lesen dennoch motivieren. Für die Schüler dürften der mediale Dialog mit Jonas' dunklem Begleiter sowie die zahlreichen unheimlichen Spuren seiner Existenz die besondere Attraktion der Lektüre darstellen. Diese Spuren zu verfolgen und sie in eine kohärente Deutung zu überführen, erfordert intensives, textnahes Lesen. ${ }^{85}$ Das Interesse der Schüler am Unheimlichen, der Wille, zu erfahren, was es mit dem Schläfer auf sich hat, ob er mit oder gegen Jonas arbeitet, führt so beinahe selbstverständlich zur genauen, man könnte sagen, kriminalistischen Lektüre. Steht hier besonders die Förderung von Lese- und Verstehenskompetenz im Vordergrund, so bietet der Text darüber hinaus gerade hinsichtlich der Identitätsorientierung Ansatzpunkte, um weiterführendes literarisches Lernen anzubahnen.

Trotz der zahlreichen Irritationen und Schwierigkeiten verfügt der Roman über ein beachtliches Identifikationspotential. Jonas ist eine Figur aus dem Hier und Jetzt. Er lebt in einer Welt der Medien, die den Schülern gut vertraut ist. Sich in Jonas Situation zu versetzen, zu diskutieren, welche Entscheidungen man an seiner Stelle treffen würde, all dies sind Fragen, die sich Schüler ganz automatisch stellen und die im literarischen Gespräch gezielt zu thematisieren sind. Gerade der für Schüler auf den ersten Blick sicherlich nicht sonderlich befriedigende Schluss des Romans - Jonas entzieht sich durch Suizid der psychisch zermürbenden Isolation in einer menschenleeren Welt - kann solche Diskussionen vertiefen und die Wahrnehmung des Textes schärfen. Die zahlreichen offengebliebenen Fragen bieten dann die Möglichkeit, auch die philosophische Dimension des Textes schrittweise zu erschließen. Die Arbeit der Nacht lädt dazu ein, sich lustvoll auf die Unabschließbarkeit des Sinnbildungsprozesses und die Fremdheit der angebotenen Perspektive einzulassen. Man kann diese Offen-

85 Vgl. Kämper-van den Boogaart 2010. 
heit als Problem, aber auch als Herausforderung begreifen, die sich in verschiedenen Anschlusskommunikationen fruchtbar machen lässt.

Den Schülern stehen für ihre Auseinandersetzung mit dem Roman verschiedene mediale Ressourcen zur Verfügung. Beispielsweise können sie den Autor auf dessen Homepage in einem Podcast auf seinem Spaziergang zu den Handlungsorten des Romans in Wien begleiten. Die Möglichkeiten literarischer Bildung werden auf diese Weise um die auditive Dimension ästhetischer Erfahrung erweitert. Ein Blick auf die Internetplattform Youtube zeigt, dass der Roman bereits einige Leser animiert hat, ihre Lektüreeindrücke filmisch zu gestalten. Aus solchen Angeboten lassen sich für eine vertiefende produktionsorientierte Arbeit am Text wichtige Impulse gewinnen. Überhaupt kann am Beispiel von Die Arbeit der Nacht verdeutlicht werden, welche Chancen die reflektierte Nutzung des Internets für den Literaturunterricht bietet. Schnell können die Schüler mit der Hilfe von Internet-Rezensionen oder Blogbeiträgen einen Überblick über Themen und Deutungsansätze gewinnen und diese mit den Ergebnissen ihrer eigenen Arbeit vergleichen. Vor allem aber können sie sich interaktiv an literaturbezogenen Diskussionen beteiligen. Es sind hier zahlreiche Möglichkeiten denkbar, die über die eingespielten Arbeitsformen des Literaturunterrichts hinausgehen. Der Erwerb von Medienkompetenz ließe sich so effektiv mit dem literarischen Lernen verbinden. ${ }^{86}$

Ist das Interesse der Schüler für die unterschiedlichen Facetten des anspruchsvollen Romans erst einmal geweckt, lässt sich sinnvoll ein historischer Exkurs anschließen, der die Spuren des Denkbildes der Isolation in die Literaturgeschichte verfolgt. $\mathrm{Zu}$ welchen Lösungen gelangen andere Werke, die ähnliche Themen und Situationen gestalten? Die Reihe der möglichen Texte kann in exemplarischer Weise über Arno Schmidts Schwarze Spiegel (1951), Herbert Rosendorfers Großes Solo für Anton (1976), Marlen Haushofers Die Wand (1963) bis hin zu Defoes Robinson Crusoe und den frühneuzeitlichen Dystopien geführt werden. Ergänzend herangezogen werden können andere Medien wie Hörbücher und Literaturverfilmungen, durch die sich die Wahrnehmung des Themas erweitern lässt. Julian Pölslers aktuelle Verfilmung (2012) von Haushofers Isolationsroman Die Wand eignet sich gut, um den Schülern einen ganz anderen, gendersensiblen Blick auf die in Die Arbeit der Nacht gestaltete Situation zu vermitteln. Die mittlerweile recht rege Forschung zu Glavinics Roman hat darüber hinaus Ergebnisse geliefert, die zusätzliche Anregungen für den Literaturunterricht bieten. So kann man etwa exemplarisch nach der Stellung des Textes in der Tradition des erkenntnistheoretischen Scheiterns fragen, wie sie Marta

86 Vgl. Frederking / Krommer / Maiwald 2008; Krommer / Dreier 2010; Barsch 2006; Groeben / Hurrelmann (Hrsg.) 2002; Tulodziecki / Bardo / Graf 2010. 
Famula skizziert hat und intertextuelle Verweise zwischen Glavinics Roman und Texten von Dürrenmatt und Kafka verfolgen. ${ }^{87}$ Auch aus Daniela Hempens Untersuchung zur Bedeutung der Natur-Kultur-Dichotomie ließe sich ein Beobachtungsschwerpunkt gewinnen. ${ }^{88}$ Eine weitere Anregung liefert Maria KublitzKramer. In ihrer Studie fokussiert sie die alltäglichen Praktiken der menschlichen Existenz - hier das Essen - unter den Bedingungen der Isolation. ${ }^{89}$ Diese Perspektive auf weitere im Texte gestaltete Elementarpraktiken auszudehnen, kann den Schülern wichtige Hilfestellungen bei der Vertiefung des Textverstehens bieten.

Die bis hierher beschriebenen Möglichkeiten, die Historizität menschlicher Elementarerfahrungen am Beispiel von Die Arbeit der Nacht herauszustellen, kann für die Schüler einen Anlass bieten, sich im Prozess der individuellen Identitätsarbeit zu den geschichtlichen Entwürfen in Beziehung zu setzen und ihre Eindrücke, Gedanken und Ideen im literarischen Gespräch oder im Rahmen verschiedener Schreibaufträge zum Ausdruck zu bringen. Die Identitätsorientierung des Unterrichts vollzieht sich hierbei nicht durch die Fokussierung auf das Eigene und Vertraute, sondern das Andere und das Fremde werden gezielt ins Spiel gebracht. In einer Welt, in der das Vertraute an Selbstverständlichkeit verliert, wird die Auseinandersetzung mit dem Fremden zur konstitutiven Bedingung der Identitätsarbeit. In diesem Sinne ließe sich mit Hilfe historischer Reihenbildungen, ${ }^{90}$ die vom Hier und Jetzt in die Geschichte führen, ein Literaturunterricht denken, der die Gegenwart im Blick hat, ohne der Gefahr ausgesetzt zu sein, vor der Christian Dawidowski so eindringlich warnt:

Eine Integration der literarischen Gegenwart in ihrer vor allem gattungsspezifischen Breite kann so nur über eine Vernetzung mit bestehenden curricularen Strukturen und Inhalten gelingen. Bleibt die Gegenwartsliteratur ein Solitär, der weder epochenspezifisch, noch gattungstypologisch noch über die literarische Motivik an Bestehendes angebunden wird, kann sie ausgetauscht und weggekürzt werden. (2012: 13)

Nach der ausführlicheren literatur- und mediendidaktischen Reflexion von Die Arbeit der Nacht, sollen abschließend zumindest noch wenige Stichworte zu einigen anderen Romanen Glavinics ergänzt werden. ${ }^{91}$

Von ähnlicher thematischer Attraktivität wie Die Arbeit der Nacht sollte für Schüler der Sek. II Glavinics Roman Das Leben der Wünsche (2009) sein. Drei

87 Marta 2009.

88 Hempen 2011.

89 Kublitz-Kramer 2008.

90 Vgl. aus einer medienintegrativen Perspektive auch Abraham 2012, S. $192 \mathrm{ff}$.

91 Vgl. hierzu auch die weiteren literatur- und mediendidaktischen Beiträge im vorliegenden Band. 
Wünsche werden der Hauptfigur Jonas, einem 35jähriger Werbetexter mit Frau, Kind und heimlicher Affäre, angeboten. Nach anfänglicher Skepsis lässt er sich auf das Spiel der Wünsche ein, wodurch sein Leben mehr und mehr aus den Fugen gerät. Jonas wird im Verlauf der Handlung zum Spielball einer nicht mehr zu stoppenden zerstörerischen Schicksalsmaschinerie. Das schon für Die Arbeit der Nacht empfohlene Verfahren der historisch-intertextuellen Reihenbildung ließe sich ebenso auf diesen Roman übertragen. Als Bezugs- und Vergleichstexte können klassische Volksmärchen, Goethes Faust, aber z.B. auch Erich Kästner Erzählung Das Märchen vom Glück herangezogen werden. In besonderer Weise lädt Das Leben der Wünsche zum identifikatorischen Lesen ein. Das Thema des ungebändigten Wünschens verleitet geradezu zum symbolischen Probehandeln und zur kritischen Diskussion. Zur Lesemotivation beitragen kann weiterhin die Auseinandersetzung mit dem aufwendig produzierten Buchtrailer, der auf Youtube abrufbar ist. Hierbei handelt es sich um eine neuartige Form des literarischen Kurzfilms, die für den Literaturunterricht über ein außerordentliches und bislang noch kaum ausgeschöpftes didaktisches Potential verfügt.

Der eingangs bereits angesprochene Literaturbetriebsroman Das bin doch ich eröffnet den Schülern einen spannenden Blick hinter die Kulissen des Literaturbetriebs. Es dürfte interessant sein, die Schüler ihre Vorstellungen von Autorschaft mit den Eindrücken aus der Lektüre von Das bin doch ich vergleichen zu lassen. Mit gezielt ausgewählten Passagen aus anderen Texten - z.B. aus Goethes Dichtung und Wahrheit - kann die Arbeit begleitet und gelenkt werden. Aus dieser kontextualisierenden Lektüre lässt sich die Aufmerksamkeit auf das für den Roman konzeptuell zentrale Verhältnis von Fiktionalität und Faktizität zuspitzen.

Der Roman Wie man leben soll (2006) spielt auf ironische Weise mit dem Genre der Adoleszenzliteratur. Diese ,Familienähnlichkeit' empfiehlt den Text als ergänzende Lektüre im Zusammenhang mit den im Deutschunterricht viel gelesenen klassischen Gattungsvertretern. ${ }^{92}$ Symbolisch gerahmt durch zwei der großen Katastrophen der amerikanischen Raumfahrt - der Explosion des Space Shuttles Challenger am 28. Januar 1986 sowie der Columbia am 1. Februar 2003 - wird die Entwicklungsgeschichte des übergewichtigen Karl Kolostrum erzählt. Karl ist ein Antiheld, der mit den Figuren der klassischen Adoleszenztexte wenig gemein hat. Schule, erste Liebe, Familienkonflikte, Sinnsuche, Studium, berufliche Orientierung - dies sind die Themen, über die erzählt wird. Durchzogen ist die Handlung von grotesken Todesfällen, in die Karl auf bizarre Weise verwickelt ist. Von den zahlreichen pikant-irritierenden Passagen, die seinen Weg ins Erwachsenenalter pflastern, sollte man sich nicht abschrecken 
lassen. Wenn die Überzeichnungen von Figuren und Situationen bewusst als literarische Gestaltungsmittel wahrgenommen werden, ist bereits ein wichtiger analytischer Schritt getan. Die im Text vorgeführten und durch die vielfach anzitierte psychologische Ratgeberliteratur lustvoll konterkarierten Weltbilder dürften die Schüler zur kritischen Reflexion anregen. In Verbindung mit der Verfilmung kann die Frage „Wer bin ich und wer möchte ich sein?“ zur motivierenden Grundlage einer identitätsorientierten Unterrichtssequenz gemacht werden.

Mit Verfilmung und Hörspiel bietet der Kriminalroman Der Kameramörder vielfältige Ansatzpunkte für einen medienintegrativen Literaturunterricht. Erzählt werden im Protokollstil die Ereignisse rund um die Ausstrahlung eines Videos, das die erzwungene Selbsttötung entführter Kinder zeigt. Zwei befreundete Paare verbringen das Osterwochenende miteinander. Unter ihnen befindet sich auch der Ich-Erzähler, aus dessen Perspektive das Geschehen rückblickend berichtet wird. Im Fernsehen verfolgen sie die Berichte über eine Kindesentführung und die hitzige Diskussion um die bevorstehende Ausstrahlung des besagten Videos. Erst ganz am Schluss stellt sich heraus, dass es sich bei dem IchErzähler um den ,Kameramörder' handelt. Auch dieser Text erfordert eine ,kriminalistische“ Lektüre. Das überraschende Ende lädt zur vertieften Relektüre und dem analytischen Aufspüren von Leerstellen ein. Besonders angesprochen und gefordert wird bei der Behandlung dieses Textes sicherlich das kritische Bewusstsein der Schüler. Neben der spannenden Kriminalgeschichte steht die Herausforderung, Stellung zum aktuellen Mediendiskurs zu beziehen. Die Frage, wo das Böse zu Hause ist, wird ergänzt durch die moralische Problematisierung der modernen Informationsmedien. Diese beiden Perspektiven sind mit der genauen Lektüre des Textes auszubalancieren.

Möchte man abschließend einen roten Faden im Werk Glavinics benennen, der seine Texte für den Deutschunterricht interessant gestaltet, so ist neben dem großen thematischen Spektrum und der Konzentration auf irritierende Identitätsentwürfe sicherlich die dominante Präsenz der Medien hervorzuheben. Die Medien sind als Objekte nicht nur Bestandteil der fiktionalen Welten, sie werden wie in Die Arbeit der Nacht, im Kameramörder oder auch in Lisa gewissermaßen selbst zu unheimlichen Handelnden. Dass hier eine besondere Attraktivität für den Deutschunterricht zu sehen ist, liegt auf der Hand. Aber Thomas Glavinic ist nicht nur ein Autor, der über die Medien schreibt. Als Autor ist er selbst mit den Medien eine virtuelle Symbiose eingegangen. Seine Präsenz auf der eigenen Homepage, auf Facebook oder Youtube vermag die Distanz zum Autor zumindest ansatzweise zu überbrücken, mit der sich Leser im Rahmen der literarischen Kommunikation doch sonst konfrontiert sehen. Für den Literaturunterricht ergeben sich dadurch spannende Perspektiven. Das Prinzip der Abwesenheit des Autors lässt sich hier aufbrechen. Auch das mag ein Stück weit unheim- 
lich sein. Für die Schüler jedenfalls stellt sich die interpretationstheoretisch problematische Frage „Was will uns der Autor sagen?“ in ganz neuer Weise und in diesem Fall - sogar mit der potentiellen Aussicht auf eine Antwort. Gerade der Blick auf die Selbstpräsentationen des Autors vermag einen wichtigen Berührungspunkt mit den Erkenntnisinteressen der Gegenwartsliteraturforschung aufzuzeigen.

Die Beiträge des Bandes setzen auf je eigene Weise die Suche nach den Schnittstellen zwischen den Teildisziplinen fort. Es ist nicht ausgeschlossen, dass Literatur- bzw. Mediendidaktik und Literaturwissenschaft in ihrem geteilten Interesse an der Gegenwartsliteratur etwas voneinander lernen.

\section{Literaturverzeichnis}

Abraham, Ulf u. Christoph Launer (Hrsg.): Weltwissen erlesen. Literarisches Lernen im fächerverbindenden Unterricht. Baltmannsweiler: Schneider 2002.

Abraham, Ulf u. Matthis Kepser: Literaturdidaktik Deutsch. Eine Einführung, 3. neu bearb. u. erw. Aufl. Berlin: Erich Schmidt 2009.

Abraham, Ulf, Albert Bremerich-Vos, Volker Frederking und Petra Wieler (Hrsg.): Deutschdidaktik und Deutschunterricht nach PISA. Freiburg im Breisgau: Fillibach 2003.

Abraham, Ulf: Fantastik in Literatur und Film. Eine Einführung für Schule und Hochschule. Berlin: Erich Schmidt 2012.

Rauch, Marja: Renaissance des Phantastischen. Aktuelle Tendenzen in Literatur und Film. In: Literatur im Unterricht 1 (2011), S. 51-62.

Allkemper, Alo, Norbert Otto Eke, Hartmut Steinecke (Hrsg.): Poetologisch-poetische Interventionen: Gegenwartsliteratur schreiben. München: Fink 2012.

Assmann, David-Christopher: Das bin nicht ich. Thomas Glavinics Literaturbetriebs-Szene. In: Thomas Wegmann u. Norbert Christian Wolf (Hrsg.): „High“ und „low“: Zur Interferenz von Hoch- und Populärkultur in der Gegenwartsliteratur, Berlin/New York: de Gruyter 2011, S. 121-141.

Bark, Joachim und Jürgen Förster (Hrsg.): Schlüsseltexte zur neuen Lesepraxis. Stuttgart: Klett 2000.

Barsch, Achim: Mediendidaktik Deutsch, Paderborn: Schöningh 2006.

Bartl, Andrea, Jörn Glasenapp u. Iris Hermann (Hrsg.): Zwischen Alptraum und Glück. Thomas Glavinics Vermessung der Gegenwart. Göttingen: Wallstein 2014.

Bierwirth, Maik, Anja Johannsen, Mirna Zeman (Hrsg.): Doing Contemporary Literature. Praktiken, Wertungen, Automatismen. München: Fink 2012.

Bluhm, Lothar: Gegenwartsliteratur. In: Burdorf, Dieter, Christoph Fasbender u. Burkhard Moennighoff (Hrsg.): Metzler Lexikon Literatur, 3. völlig neu bearb. Aufl., Stuttgart: Metzler 2007, S. 267.

Bogdal, Klaus-Michael: Deutschland sucht den Super-Autor. Über die Chancen der Gegenwartsliteratur in der Mediengesellschaft. In: Kammler, Clemens (Hrsg.): Deutschsprachige Gegenwartsliteratur seit 1989, S. 85-97. 
Bogdal, Klaus-Michael: Mein ganz persönlicher Duft. Das Parfum, die Didaktik und der Deutschunterricht, in: Diskussion Deutsch 24. Jg. (1993), S. 124-133.

Borchmeyer, Dieter und Helmuth Kiesel (Hrsg.): Der Ernstfall. Martin Walsers Tod eines Kritikers. Hamburg: Hoffmann und Campe 2003.

Bossinade, Johanna: Poststrukturalistische Literaturtheorie. Stuttgart/Weimar: Metzler 2000.

Braun, Michael: Die deutsche Gegenwartsliteratur. Köln u.a.: Böhlau 2010.

Brodowsky, Paul u. Thomas Klupp (Hrsg.): Wie über Gegenwart sprechen? Methoden einer Gegenwartsliteraturwissenschaft. Frankfurt a.M./New York: Peter Lang 2011.

Brüggemann, Jörn: Literarizität und Geschichte als literaturdidaktisches Problem. Eine Studie am Beispiel des Mittelalters. Frankfurt a.M./New York: Peter Lang 2008.

Burtscher, Sabine: Auswahlkriterien für Gegenwartsliteratur im Deutschunterricht. Ein Beitrag zur Revision der Kanondiskussion. In: Literatur im Unterricht 1 (2003), S. 3-20.

Dawidowski, Christian und Dieter Wrobel (Hrsg.): Interkultureller Literaturunterricht. Baltmannsweiler: Schneider 2006 (= Diskussionsforum Deutsch 22).

Dawidowski, Christian: Literaturunterricht zwischen Zentralisierung und Innovation. Wo bleibt die Gegenwartsliteratur? In: ders.: Gegenwartsliteratur und Postmoderne im Literaturunterricht. Baltmannsweiler: Schneider 2012, S. 2-15.

Dreier, Ricarda: Literatur der 90er- Jahre in der Sekundarstufe II: Judith Hermann, Benjamin von Stuckrad-Barre und Peter Stamm. Baltmannsweiler: Schneider 2005.

Erb, Andreas (Hrsg.): Baustelle Gegenwartsliteratur. Die neunziger Jahre, Opladen: VS 1998.

Famula, Marta: Gleichnisse des erkenntnistheoretischen Scheiterns. Thomas Glavinics Roman Die Arbeit der Nacht in der Tradition des labyrinthischen Erzählens bei Franz Kafka und Friedrich Dürrenmatt. In: Andrea Bartl (Hrsg.): Transitträume. Beiträge zur deutschsprachigen Gegenwartsliteratur. Augsburg: Wißner 2009, S. 103-122.

Fingerhut, Karlheinz: Die folgenlose Literatur und der pädagogische Wahn. Deutschdidaktik, Literaturunterricht und die Gegenwartsliteratur. In: Norbert Oellers (Hrsg.): Germanistik und Deutschunterricht im Zeichen der Technologie. Selbstbestimmung und Anpassung. Vorträge des deutschen Germanistentages. Tübingen: Niemeyer 1988, S. 3-22.

Frank, Horst-Joachim: Geschichte des Deutschunterrichts. Von den Anfängen bis 1945. München: Dt. Taschenbuchverlag 1977.

Frederking, Volker, Axel Krommer u. Klaus Maiwald: Mediendidaktik Deutsch. Eine Einführung. Berlin: Erich Schmidt 2008.

Frederking, Volker: Identitätsorientierter Literaturunterricht. In: ders., Hans-Werner Huneke, Axel Krommer u. Christel Meier (Hrsg.): Taschenbuch des Deutschunterrichts, Bd. 2: Literatur- und Mediendidaktik, Baltmannsweiler: Schneider 2010, S. 414-452, hier S. 423.

Friedrich, Hans-Edwin (Hrsg.): Literaturskandale. Frankfurt a.M./Berlin: Peter Lang 2009.

Glavinic, Thomas: Carl Haffners Liebe zum Unentschieden. Berlin: Dt. Taschenbuchverlag 1998.

Glavinic, Thomas: Das größere Wunder. München: Hanser 2013.

Glavinic, Thomas: Das Leben der Wünsche. München: Hanser 2009.

Glavinic, Thomas: Der Kameramörder. Berlin: Dt. Taschenbuchverlag 2001.

Glavinic, Thomas: Die Arbeit der Nacht. München: Hanser 2006.

Glavinic, Thomas: Lisa. München: Hanser 2011.

Glavinic, Thomas: Unterwegs im Namen des Herrn. München: Hanser 2011.

Glavinic, Thomas: Meine Schreibmaschine und ich. Hanser: München 2014. 
Glavinic, Thomas: Wie man leben soll. München: Hanser 2004.

Graf, Werner: Der Sinn des Lesens. Modi der literarischen Rezeptionskompetenz, Münster: Lit 2004.

Groeben, Norbert u. Bettina Hurrelmann (Hrsg.): Medienkompetenz. Voraussetzungen, Dimensionen, Funktionen. Weihnheim/München: Juventa 2002.

Hegewald, Wolfgang: Fegefeuernachmittag. Mein Leben. Von ihm selbst erzählt. Berlin: Matthes \& Seitz 2009.

Heidi Rösch (Hrsg.): Literarischen Bildung im kompetenzorientierten Deutschunterricht. Freiburg im Breisgau: Fillibach 2010.

Hellberg, Wolf Dieter: Lektüreschlüssel zu Daniel Kehlmann: Die Vermessung der Welt. Stuttgart: Reclam 2012.

Hempen, Daniela: Wolfsvieh, Flügelbär und König Etzels Grab...: Ungezähmte Natur als falscher locus terribilis in Thomas Glavinics Endzeitroman Die Arbeit der Nacht. In: Neophilologus 95 (2011), S. 291-303.

Herwig, Henriette: Identität und Fremdverstehen in interaktionistischer und literaturdidaktischer Sicht, in: Spinner, Kaspar H. (Hrsg.): Identität und Deutschunterricht. Göttingen: Niemeyer 1980, S. 15-32.

Hinkerohe, Frank: Martin Walsers Roman Tod eines Kritikers in der Diskussion der deutschen Literaturkritik. Duisburg: WiKu-Verl. 2006.

Hofer, Daniel: Ein Literaturskandal, wie er im Buche steht. Zu Vorgeschichte, Missverständnissen und medialem Antisemitismusdiskurs rund um Martin Walsers Roman Tod eines Kritikers. Berlin/Wien: LIT 2007.

Horstkotte, Silke / Herrmann, Leonhard (Hrsg.): Poetiken der Gegenwart. Deutschsprachige Romane nach 2000. Berlin/New York: de Gruyter 2013.

Huber, Martin u. Albert Meier (Hrsg.): Bestseller des 21. Jahrhunderts. Göttingen 2010 (= MDGV).

Irmgard Nickel-Bacon u. Dieter Wrobel: Lesekultur. In: Praxis Deutsch 231 (2011), S. 1-8.

Kammler, Clemens (Hrsg.): Literarische Kompetenzen - Standards im Literaturunterricht. Modelle für die Primar- und Sekundarstufe, Seelze: Kallmeyer 2006.

Kammler, Clemens u. Volker Surmann: Sind Deutschlehrer experimentierfreudig? In: Der Deutschunterricht 6 (2000), S. 92-96.

Kammler, Clemens: Deutschsprachige Literatur seit 1989/90. Ein Rückblick. In: Deutschsprachige Gegenwartsliteratur seit 1989, S. 13-37.

Kammler, Clemens: Die deutschsprachige Gegenwartsliteratur. Ein Problemfall der literarischen Sozialisation. In: Zeitschrift für Literaturwissenschaft und Linguistik 31 (2001), H. 124, S. 140-150.

Kammler, Clemens: Gegenwartsliteratur im Unterricht. In: Bogdal, Klaus-Michael und Hermann Korte (Hrsg.): Grundzüge der Literaturdidaktik. München: Dt. Taschenbuchverlag 2002, S. 166-177.

Kammler, Clemens: Gegenwartslücken. Anmerkungen zu einem Defizit des Literaturunterrichts. In: Baustelle Gegenwartsliteratur, S. 186-202.

Kammler, Clemens: Plädoyer für das Experiment. Deutschunterricht und Gegenwartsliteratur in der Schule. In: Diskussion Deutsch 26 (1995), H. 142. S. 127-135. 
Kammler, Clemens: Was kommt nach Dürrenmatt und Frisch? Plädoyer für einen anderen Umgang mit Gegenwartsliteratur in der Schule. In: Diskussion Deutsch 26 (1995), H. 142, S. 127-135.

Kammler, Clemens: Neue Literaturtheorien und Unterrichtspraxis. Positionen und Modelle. Baltmannsweiler: Schneider 2000.

Kämper-van den Boogaart, Michael: Gegenwartsliteratur und schulischer Lektürekanon. Aspekte einer ambivalenten Beziehung. In: Clemens Kammler und Torsten Pflugmacher (Hrsg.): Deutschsprachige Gegenwartsliteratur seit 1989. Zwischenbilanzen - Analyse Vermittlungsperspektiven. Heidelberg: Synchron 2004, S. 251-263.

Kämper-van den Boorgaart, Michael: Textnahes Lesen. In: Taschenbuch des Deutschunterrichts, S. 271-283.

Keupp, Heiner u.a.: Identitätskonstruktionen. Das Patchwork der Identitäten in der Spätmoderne. 4. Aufl. Reinbek: Rowohlt 2008.

Köhler, Andrea (Hrsg.): Maulhelden und Königskinder. Zur Debatte über deutschsprachige Gegenwartsliteratur, Leipzig: Reclam 1998.

Kreft, Jürgen: Grundprobleme der Literaturdidaktik. Eine Fachdidaktik im Konzept sozialer und individueller Entwicklung und Geschichte. Heidelberg: Quelle \& Meyer 1977.

Krommer, Axel u. Ricarda Dreier: Medienkompetenz im symmedialen Deutschunterricht. In: Taschenbuch des Deutschunterrichts, S. 669-691.

Krumrey, Birgitta: Experimentierfreude und „Spieltrieb“. Thomas Glavinics Kriminalroman Lisa. In: Eichhorn, Kristin (Hrsg.) Neuer Ernst in der Literatur? Schreibpraktiken in deutschsprachigen Romanen der Gegenwart, Frankfurt a.M./Berlin u.a.: Peter Lang 2014, S. 17-33.

Kublitz-Kramer, Maria: Einsame Mahlzeiten. Alleinessende in Marlen Haushofers Die Wand und Thomas Glavinic' Die Arbeit der Nacht. In: Claudia Lillge u. Anne-Rosa Meyer (Hrsg.): Interkulturelle Mahlzeiten. Kulinarische Begegnungen und Kontraste in der Literatur. Bielefeld: Transcript 2008, S. 277-293.

Köhler, Andrea / Moritz, Rainer (Hrsg.): Maulhelden und Königskinder. Zur Debatte über die deutschsprachige Gegenwartsliteratur. Leipzig: Reclam 1998.

Kyora, Sabine (Hrsg.): Subjektform Autor. Autorschaftsinszenierungen als Praktiken der Subjektivierung. Bielefeld: Transcript 2014.

Lange, Günter (Hrsg.): Kinder- und Jugendliteratur der Gegenwart. Ein Handbuch. Baltmannsweiler: Schneider 2012.

Gansel, Carsten: Moderne Kinder- und Jugendliteratur. Vorschläge für einen kompetenzorientierten Unterricht. 4. überarb. Aufl. Berlin: Klett 2010.

Lange, Günter: Erwachsen werden. Jugendliterarische Adoleszenzromane im Deutschunterricht. 3. überarb. u. veränderte Aufl. Baltmannsweiler: Schneider 2012.

Modick, Klaus: Bestseller. Frankfurt a.M.: Piper 2006.

Müller-Michaels, Harro: Denkbilder. Zu Geschichte und didaktischem Nutzen einer literarischen Kategorie. In: Deutschunterricht 49 (1996), S. 114-122.

Nadolny, Arnd: Die Vermessung der Welt von Daniel Kehlmann: Textanalyse und Interpretation mit ausführlicher Inhaltsangabe und Abituraufgaben mit Lösungen. Hollfeld: Bange 2012.

Neuhaus, Stefan und Johann Holzner (Hrsg.): Literatur als Skandal. Fälle - Funktionen - Folgen. Göttingen: Vandenhoeck \& Ruprecht 2007. 
Neuhaus, Stefan: „Literatur und Identität. Zur Relevanz der Literaturwissenschaft“, in: Magerski, Christine u. Svjetlan Lacko Vidulić (Hrsg.): Literaturwissenschaft im Wandel. Aspekte theoretischer und fachlicher Neuorganisation. Wiesbaden: VS 2009, S. 81-95.

Neuhaus, Stefan: Martin Walsers Roman Tod eines Kritikers und seine Vorgeschichte(n), Oldenburg 2004.

Orths, Markus: Lehrerzimmer. Frankfurt a.M.: Schöffling 2003.

Paefgen, Elisabeth K.: Einführung in die Literaturdidaktik. 2. Aktual. u. erw. Aufl. Stuttgart/Weimar: Metzler 2006.

Pfäfflin, Sabine: Auswahlkriterien für Gegenwartsliteratur im Deutschunterricht. 2. korr. u. erw. Aufl. Baltmannsweiler: Schneider 2010.

Reckwitz, Andreas: Das hybride Subjekt. Eine Theorie der Subjektkulturen von der bürgerlichen Moderne zur Postmoderne. Weilerswist: Velbrück 2006.

Rösch, Heidi (Hrsg.): Kompetenzen im Deutschunterricht. 2. überarb. u. erw. Aufl., Frankfurt a.M.: Peter Lang 2008.

Saupe, Anja: Standards und Kompetenzmodelle in literatur- und sprachdidaktischer Perspektive. Eine Zwischenbilanz. In: Didaktik Deutsch 23 (2007), S. 134-141.

Schalansky, Judith: Hals der Giraffe. Ein Bildungsroman. Berlin: Suhrkamp 2011.

Schönbrunn, Walter: Die Not des Literaturunterrichts in der großstädtischen Schule. In: Die Erziehung 4 (1929), S. 252-259.

Schumacher, Eckhard: Gerade eben jetzt. Schreibweisen der Gegenwart. Frankfurt a.M.: Suhrkamp 2011.

Sekretariat der Ständigen Konferenz der Kultusminister der Länder in der Bundesrepublik Deutschland (Hrsg.): Bildungsstandards im Fach Deutsch für den mittleren Schulabschluss, München 2003, S. 6.

Spinner, Kaspar H.: Identitätsgewinnung als Aspekt des Aufsatzunterrichts. In: Identität und Deutschunterricht, S. 67-80.

Spinner, Kaspar: Kreativer Deutschunterricht. Identität - Imagination - Kognition. Seelze: Kallmeyer 2001.

Spinner, Kaspar: Literarisches Lernen (Basisartikel). In: Praxis Deutsch 200 (2006), S. 6-16. Spinner, Kaspar: Ästhetische Bildung und Literaturunterricht. In: Rieckmann, Carola / Gahn, Jessica (Hrsg.): Poesie verstehen - Literatur unterrichten. Baltmannsweiler: Schneider 2013, S. 17-35.

Standke, Jan: Das Schwierige (in) der literarischen Bildung. Kontroversentheoretische Überlegungen zur Verhandlung eines literaturdidaktischen ,Denkrahmens‘. In: Dawidowski, Christian (Hrsg.): Bildung durch Dichtung / Literarische Bildung: Bildungsdiskurse literaturvermittelnder Institutionen um 1900 und um 2000, Frankfurt a.M.: Peter Lang 2013, S. 281-301.

Standke, Jan: Symbolisches Textverstehen: die Erzählung Die Maske. In: Deutschunterricht 3 (2014a), S. 26-32.

Standke, Jan: Identitätsgefährdungen? Perspektiven literarischer Bildung am Beispiel der Romane Thomas Glavinics. In: Andrea Bartl u.a. (Hg.): Zwischen Alptraum und Glück: Thomas Glavinics Vermessungen der Gegenwart, Göttingen: Wallstein 2014b, S. 193221.

Standke, Jan (Hrsg.): Kehlmann lesen. Interpretationen und Unterrichtsmodelle für die Sek. I und II. Baltmannsweiler: Schneider (erscheint 2014c). 
Tulodziecki, Gerhard, Bardo Herzig u. Silke Graf: Medienbildung in Schule und Unterricht. Bad Heilbrunn: Klinkhardt 2010.

von Düffel, John: Goethe ruft an. Berlin: DuMont Buchverlag 2011.

Walser, Martin: Tod eines Kritikers. Frankfurt a.M.: Suhrkamp 2002.

Wintersteiner, Werner: Alte Meister - Über die Paradoxien literarischer Bildung. In: Didaktik Deutsch 30 (2011), S. 5-22.

Wrobel, Dieter: Romane von Kafka bis Kehlmann. Literarisches Lernen in der Sekundarstufe I und II. Seelze: Kallmeyer 2013.

Zabka, Thomas (Hrsg.): Schule in der neueren Kinder- und Jugendliteratur. Baltmannsweiler: Schneider 2008.

Zima, Peter V.: Das literarische Subjekt. Zwischen Spätmoderne und Postmoderne. Tübingen/Basel: Francke 2001. 